\title{
Optimizing Selective ARQ for H.264 Live Streaming: A Novel Method for Predicting Loss-Impact in Real Time
}

\author{
Michael Schier, Associate Member, IEEE, and Michael Welzl, Associate Member, IEEE
}

\begin{abstract}
This work proposes a quality-oriented, real-time capable prioritization technique for media units of H.264/AVC video streams. The derivation of estimates is based on the analysis of the macroblock partitioning, the spatial extents of temporal dependencies, and the length and strength of prediction chains existing among macroblocks, thus incorporating the expected impact of error propagation. It is demonstrated how the prioritization scheme can be beneficially integrated into live streaming systems which are characterized by tight timing constraints, with the focus on content-aware selective automatic repeat request mechanisms. Additionally, it is shown how potentially limited feedback can be used to adapt the estimation process to leverage prediction preciseness. The approach is compared against existing techniques in terms of practicability and efficiency, and tested under independent and bursty loss conditions in a wired and a wireless test setup. Moreover, the performance is examined when low-latency and constant bitrate video settings are enforced by using $x 264$ 's novel encoding feature periodic-intra-refresh. Results of both experiments and simulations indicate that the proposed technique outperforms all reference techniques in nearly all test cases, and that the video quality can be further improved by incorporating receiver feedback.
\end{abstract}

Index Terms-End-to-end distortion modeling, error propagation, H.264/AVC, live video streaming, packet loss, selective automatic repeat request (ARQ).

\section{INTRODUCTION}

$\mathbf{O}$ VER the last few years, there has been a strongly increasing interest in streaming video over packet switched networks, and it can be expected that this trend is set to continue. This phenomenon can be mainly attributed to the modernization of telecommunication networks, the resulting wide availability of high-speed internet access, the growing market of mobile entertainment, and the increasing number of web sites which offer their users high-quality multimedia content. In many of today's packet switched networks, traffic is handled in a best-effort manner, and measures that guarantee a certain quality-ofservice such as guaranteed bandwidth or limited delay are rarely in place. Consequently, video streaming systems have to cope with the drawbacks of such hardly predictable environments,

Manuscript received May 11, 2011; revised August 24, 2011; accepted November 22, 2011. Date of publication December 06, 2011; date of current version March 21, 2012. The associate editor coordinating the review of this manuscript and approving it for publication was Dr. Beatrice Pesquet-Popescu.

M. Schier is with the Institute of Computer Science, University of Innsbruck, A-6020-Innsbruck, Austria (e-mail: michael.schier@uibk.ac.at).

M. Welzl is with the Department of Informatics, University of Oslo, 0316-Oslo, Norway (e-mail: michawe@ifi.uio.no).

Digital Object Identifier 10.1109/TMM.2011.2178235 which are primarily fluctuating delay and packet loss. They have to adopt an appropriate combination of error resilience and robustness techniques to prevent, or at least mitigate, the impact of packet loss on the video quality experienced by end users.

Some media units contribute more to the quality degradation in case of loss than others, which is due to the complex structure of today's video codecs, the diversity of content to encode, and the spatio-temporal dependencies between data elements. Several techniques have been proposed to determine the expected loss-distortion of individual units or groups of media units. Mapping such estimates to network packets is usually straightforward: As an example, an H.264/MPEG-4 AVC video stream is a sequence of network abstraction layer (NAL) units, and there exists a one-to-one correspondence between NALunits and network packets when slices are dimensioned accordingly. Providing importance estimates at the packet level allows the adoption of content-aware error handling and protection mechanisms. Application scenarios encompass, amongst others, prioritized packet drop schemes [1], [2], selective automatic repeat request (ARQ) [3]-[5], unequal forward error correction (FEC) [6], [7], the exploitation of quality-of-service (QoS) capabilities of wireless networks [8], and the assignment of packets to different service levels according to their impact on video quality [9], [10].

In this work, we provide a simple categorization of existing channel distortion models in terms of computational complexity and real-time feasibility and underline the lack of mechanisms that are both accurate and real-time capable. We then discuss the accuracy and practical relevance of a novel distortion estimation approach which meets these requirements, recently proposed by us in [11] and [12], where we demonstrated that its use can lead to substantial video quality improvements in the context of rateless error protection. Here, we focus on its applicability to systems supporting selective ARQ and extend it to incorporate potentially limited receiver feedback for dynamic estimate adaptation.

We already investigated video streaming with retransmissionbased partial reliability in an earlier work [3]. However, we focused on MPEG-4 ASP which is less complex than H.264 regarding dependencies between media units; we used an earlier, less accurate version of our distortion estimation algorithm; and most importantly, we did not consider feedback for content prioritization refinement. This feature enables continuous rescheduling of pending packets in the sender's queue in compliance with timing constraints and thus incorporates the implications of a joint loss of related media units. In this work, we 
provide an exhaustive evaluation by comparing the performance of both static and feedback-aware distortion estimation schemes against selected reference schemes in multiple test scenarios characterized by different loss behavior and delay constraints.

The remainder of this paper is organized as follows: In Section II, we consider existing, frequently referenced distortion estimation techniques, and rate them with regard to precision and practicability. In Section III, we present our distortion estimation technique which operates at the level of macroblocks and explain how the partitioning of macroblocks and motion vectors can be used to deduce the loss-impact of specific media units. Section IV sketches the simplistic streaming system used to evaluate these techniques. It discusses timing issues, how feedback can be incorporated, and presents a straightforward way to combine temporal constraints and relevance of content to a joint ranking. In Section $\mathrm{V}$, we compare the performance of our approach against selected techniques of Section II under various loss conditions and explain unexpected results. We further examine the effectiveness of all techniques when applied to streams encoded using an algorithm called periodic-intra-refresh, especially suitable for low-latency streaming. Finally, Section VI concludes the paper by summarizing the main findings.

\section{Existing ChANNEl Distortion MODELS}

The availability of a scheme that predicts the per-pixel quality degradation is not compulsory for the adaptive streaming system that we will propose in Section IV, and it is sufficient to derive a ranking of media units according to their relative loss-impact. The terms distortion estimation scheme and prioritization algorithm are therefore used interchangeably in this paper. They denote both simple/intuitive and sophisticated techniques that try to precalculate the expected distortion in terms of mean-squared error (MSE).

\section{A. Lightweight Prioritization Schemes}

Probably the simplest form of video data classification is the distinction between key- and non-key frames, as, e.g., pursued in [13] and [14]. The authors argue that the loss of a key frame affects a larger number of successive frames than the loss of a non-key frame. Thus, they preferably discard low-priority frames in times of scarce channel bandwidth. A similar approach was taken by Feamster et al. [15], who distinguish between intra-coded, inter-coded and bidirectionally-coded frames and assign priorities to frames in decreasing order as just listed to perform selective retransmissions. There exist numerous publications dating up to the present that use this rather intuitive prioritization (e.g., [6], [16]), which can be mainly attributed to its ease of implementation. With video codecs that support the concept of slicing, this technique can be expanded below the frame level. As an example, one can distinguish between instantaneous decoder refresh (IDR) and non-IDR partition $\mathrm{A}, \mathrm{B}$, and $\mathrm{C}$ slices in the case of H.264, or between different groups of blocks (GOBs) in the case of MPEG-4 ASP [17]. Layers of scalable video codecs can be considered as additional classification criterion, as proposed by Fiandrotti et al. [8].
There are some application scenarios where such simple prioritization techniques are sufficient, e.g., when media units have to be assigned to two different transport service levels. However, there are other situations where a less intuitive, finer-grained prioritization mechanism can be extremely beneficial such as in systems which support adaptive reliability.

\section{B. Sophisticated Prioritization Schemes}

In contrast to computationally cheap mechanisms, pixel-based approaches estimate the expected channel-induced distortion by anticipating the receiver-side error-concealed video stream and calculating the difference between error-free and reconstructed samples per pixel. They demand for suitable models which incorporate, amongst many other factors, the error propagation between media units, the receiver's error concealment mechanism, and the channel loss behavior. The appropriate integration of channel characteristics (average packet loss rate, loss patterns, burst length, etc.) into distortion estimation models is the subject of many studies as this is a non-trivial task.

One representative is a method called recursive per-pixel end-to-end distortion estimate (ROPE), proposed by Rose et al. [18]-[20]. ROPE recursively calculates the expected distortion at the pixel level and defines two formulae that consider the error propagation in intra- and inter-coded macroblocks. However, the model demands a close knowledge of the loss rate and assumes a Bernoulli distribution. It therefore takes no bursty loss behavior into account which prohibits its deployment in certain streaming scenarios. Moreover, pixel-filtering/averaging and subpixel motion compensation operations have to be incorporated in the model. This demands cross-correlation approximation for which it is hard to come up with an effective, low-complexity algorithm. A major drawback is the large computational effort needed to derive estimates because two moments have to be tracked at every pixel. Especially if used in combination with long and complex group of pictures (GoP) structures and if bi-directional prediction is applied which demands additional terms in the recursion formulae, real-time distortion estimation becomes an infeasible task. Furthermore, memory consumption can no longer be neglected. This applies even more to models that try to mimic (possibly iterative) error concealment techniques of present decoders as pointed out in [21], which turns the originally rather simple formulae into complex derivation sequences. Schmidt et al. point out that ROPE only considers isolated packet loss and that it ignores correlations between lost media units [20]. The incorporation of all possible loss patterns, though limited by GoP boundaries, is computationally intractable due to the exponential number of possibilities. As approximation, they propose to apply a ROPE-based first-order Taylor expansion which yields a linear approximation of the expected distortion close to a reference packet loss ratio.

Li et al. also strive to estimate the video distortion, but as expectation taken over all possible channel realizations, and investigate the impact of Markov-model burst packet losses on video quality [22]. They argue that error propagation decays over time and use a sliding window algorithm to limit the number of possible loss patterns to be considered based on that assumption. 
Although the approach appears to be promising with respect to a more general view on channel loss, there are some limitations. First, Li et al. assume that each frame is packaged into one network packet, which is not realistic from a practical point of view unless only highly compressed low-resolution sequences are used. They remark that the framework can be easily extended to the case where a frame is spread over multiple packets, but they do not address the variability of spatial error propagation over multiple frame segments. Besides that, they consider the error attenuation factors of received and lost frames as constants and ignore the diversity of content with respect to temporal and spatial complexity. Finally, they assume in their model that the correct reception and decoding of I-frames can always be guaranteed. This limitation additionally demands content-aware transportation to avoid severe implications on playback continuity in real implementations.

In contrast to the previously discussed approach, Wang et al. do not model the temporal error attenuation as constant but as a function of the packet loss ratio and the proportion of intracoded macroblocks [23]. The intra block ratio is not globally fixed but individually evaluated for each frame. Several possible function designs are discussed which incorporate intra-prediction, deblocking filtering, and sub-pel motion estimation. The appropriate choice depends on the encoder's configuration and the receiver side error concealment mechanism. Similar to $\mathrm{Li}$ et al., they argue that their approach also takes slicing below the frame level into account. Yet they restrict evaluations to a one-slice-per-frame packetization scheme and derive distortion estimates on a per-frame basis in the model verification section. They also discuss the use of single frame loss distortion estimates for unequal error protection schemes by simplifying the proposed model and assuming additive distortion behavior. However, this simplification presumes that intra-rates of different frames are fairly constant and that the channel distortion in subsequent frames decays exponentially. This is only true for a limited number of sequences, encoded using uncomplex GoP structures and short reference frame prediction lists (Wang et al. focus on an IP* GoP structure and restrict predictions only to the previous frame). Furthermore, it is not a trivial task to fit model parameters for sequences, and appropriate estimation/optimization techniques are needed to obtain them.

Babich et al. point out that the frequently applied idea to model the effects of multiple losses as the superposition of multiple independent losses is not always accurate, especially in low bit-rate wireless video communication when losses are not spaced sufficiently far apart [24]. They propose three models which build on each other. These models use the mean-squared error of consecutive frames as input to estimate the distortion caused by error propagation due to channel loss. Similar to [22], they require a globally defined constant that reflects the decay of distortion over time, but they additionally incorporate the lengths of preceding bursts. This demands a precise knowledge of the loss history, and is therefore only practical, e.g., in a system that supports selective retransmission. As in most of the previously discussed approaches, only entire frame-loss is considered.

Finally, Masala et al. propose a mechanism called analysis-by-synthesis (AbS), which estimates the overall channel-distortion by simulating the complete decoder behavior for the loss of each packet individually [5], [9], [25]-[29]. Due to the approach's packet level granularity, the estimates get quite close to the actual distortion derived at the receiver side. However, the derivation procedure is computationally expensive and is hard to carry out in real time, especially when complex concealment algorithms are in place and the propagation chain is long. If sufficiently precise estimates can be obtained from the encoder as by-product (e.g., when no temporal prediction is conducted), this drawback is negligible, but in general, complexity is high and heavy precomputation is needed to render the approach suitable for stored-video scenarios. The significant computational effort can become a bottleneck especially when new content is produced (and has to be analyzed) at high rates such as with public video platforms like YouTube where thousands of videos are uploaded every day. The co-impact of multiple packet loss is modeled in an additive way as in previously discussed approaches because an exhaustive analysis of all possible loss patterns would be computationally intractable. The authors address the limited applicability caused by the large amount of computation needed with a workaround in [26]. They propose to only use $A b S$ to evaluate the distortion introduced in the current frame, while the distortion in succeeding frames is estimated by means of an error propagation model, similar to Babich et al. Although the complexity of the modified approach is heavily reduced, its generality is questionable: the model is based on the statistical analysis of several test sequences, but only one GoP structure is considered, a fixed quantization parameter of 28 is used, and plain frame-copy error concealment is assumed. In summary, this does not guarantee the model's applicability to arbitrary settings.

In conclusion, existing approaches can be roughly categorized into computationally lightweight, intuitive techniques and pixel or block based heavyweight mechanisms. The latter seem quite diverse at first glance, but do actually have very much in common: they all calculate the distortion in terms of mean-squared error between original and artificially reconstructed pixel values and estimate the spatio-temporal error propagation in similar ways - some are even almost identical in this respect.

\section{BLOCK-LEVEL NAL UNIT PRIORITIZATION}

In what follows, we describe a technique towards prioritizing media (NAL) units with respect to the distortion in video quality caused when they are lost. For the remainder of this paper, our approach is denoted by spatial complexity and motion-tracked error propagation prioritization (ScMP), and it belongs to the category of lightweight mechanisms and is applicable to realtime scenarios. However, its design is not as intuitive as corresponding approaches presented in Section II-A.

In simplified terms, video codecs aim to minimize the storage space needed by reducing spatial and temporal redundancies. The former is limited by the size of the slice the element belongs to, which implies that similarities between macroblocks which belong to the same frame but different NAL units cannot be spatially exploited. Furthermore, the inspection of intra-prediction applied within media units is of limited benefit due to 
the fact that only the loss of entire NAL units (network packets) is considered and video decoders are generally not able to cope with bit errors between resynchronization points. Therefore, the proposed distortion estimation scheme mainly bases its estimates on the analysis of temporal dependencies. The relevance of content regarding video quality is calculated at the level of macroblocks. To obtain estimates for specific segments such as slices, the mean over all distortion estimates $\Psi_{\mathrm{ScMP}}\left(m_{i}\right)$ of macroblocks $m_{i}$ these regions contain is derived. $\Psi_{\mathrm{ScMP}}$ is the weighted sum of three sub-prioritizations, as defined in (1), in which $\sum_{j} \omega_{j}=1$ :

$$
\Psi_{\mathrm{ScMP}}(m)=\sum_{j \in\{\text { type,dep }, \mathrm{mv}\}} \omega_{j} \cdot \Psi_{j}(m) \in[0 ; 1] .
$$

\section{A. Design of $\Psi_{\text {type }}$}

The value of $\Psi_{\text {type }}(m)$ essentially reflects the partitioning of macroblock $m$. As defined by the H.264 standard [30], macroblocks can be divided into $16 \times 16,16 \times 8,8 \times 16$, and $8 \times 8$ partitions, in which the latter can be further subdivided into four differently shaped sub-partitions. At encoding time, the encoder decides which partition mode to use based on the spatial complexity of the source frames and the position of regions suitable for inter prediction within reference frame candidates. Selecting the appropriate size of an inter prediction partition is a tradeoff between the quantity of data needed to represent motion vectors and the coding gain provided by using motion compensation with smaller blocks.

To quantify the selected modes in terms of introduced distortion in case of loss, we define a lookup-table that encompasses 22 entries which are disjoint subsets of macroblock types as defined in tables 7.11-7.14 and 7.17-7.18 of the H.264 standard [30]. These values were derived by an iterative tuning process, in which they were continuously adjusted based on test results of loss simulations, measuring the videos' quality distortion in the current frame only, and deliberately neglecting the error propagation over time. This process was carried out twice using $x 264^{1}$ and $J M^{2}$ as software encoders, respectively. As expected, the two obtained tables were not identical as encoder decision making is not covered by the standard. However, the table values showed remarkable similarities, and there was the recognizable trend that distortion positively correlates with the number of partitions of a macroblock. In other words, it can be expected that the loss of a structurally simple macroblock (e.g., that contains two $8 \times 16$ partitions) causes on average a lower quality degradation than the loss of a structurally complex macroblock (e.g., one with a two-digit number of partitions). Although by only considering two different encoders we cannot claim that this approach is truly generic, we believe that the relationship between block size and loss impact also holds for other, reasonably efficient encoder implementations. Additionally, we refer to [31] and [32] where the authors came to similar conclusions. Based on this assumption, we define $\Psi_{\text {type }}(m)$ by mapping the type of a macroblock $m$ to the appropriately scaled mean value of the respective entries from both tables.

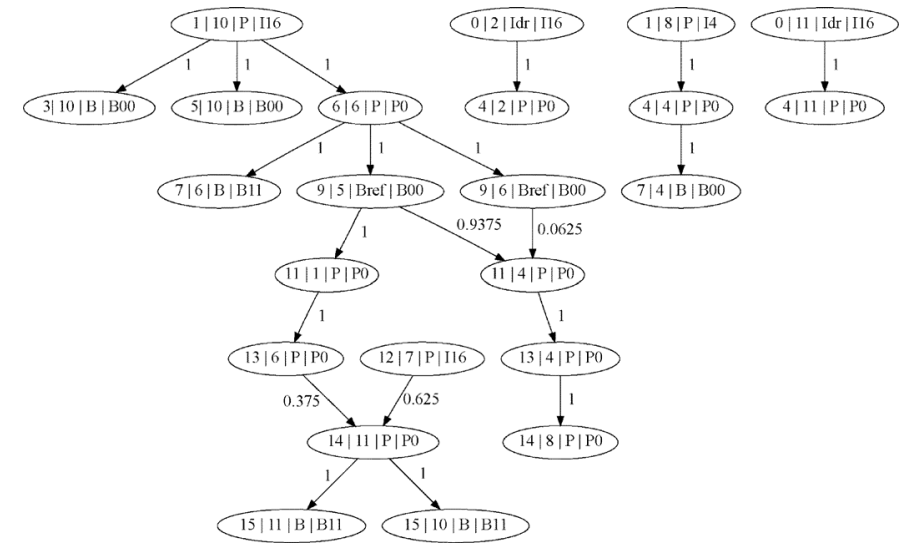

Fig. 1. Temporal dependency graph of a $48 \times 32$ test pattern sequence, heavily reduced for improved clarity. Edges have weights associated and the node labels are to be read as "frameDecodingTimestamp macroblockIndex frameType macroblockType".

\section{B. Design of $\Psi_{\mathrm{dep}}$}

$\Psi_{\text {dep }}$ reflects the temporal error propagation with respect to prediction dependencies between media units. Its derivation is performed by constructing an inverted dependency graph and weighting edges according to the sizes of affected areas. For illustration purposes, Fig. 1 depicts an exemplary, heavily reduced instance of such a graph. By studying dependency graphs, some universal properties come to light: they are acyclic due to decoding timestamps' strict monotonicity, and the length of paths is limited by the size of the current GoP (in the case of a closed-GoP structure).

Based on these observations, we can recursively compute the function $\delta_{\mathrm{dep}}(m)$ as defined in (2) and (3):

$$
\begin{aligned}
\delta_{\mathrm{dep}}(m) & =1+\sum_{v \in \mathrm{MV}(m)} \delta_{\mathrm{dep}}^{\prime}(v) \\
\delta_{\mathrm{dep}}^{\prime}(v) & =\sum_{i} \delta_{\mathrm{dep}}\left(m_{i}\right) \cdot \alpha_{i}(v) .
\end{aligned}
$$

$\delta_{\text {dep }}(m)$ reflects the cumulative size of temporally dependent areas, possibly being distributed over many frames. $\mathrm{MV}(m)$ denotes the set of forward and backward motion vectors associated with macroblock $m$, and $\delta_{\text {dep }}^{\prime}(v)$, defined in (3), reflects the sum of areas that depend on the region which $v$ points to. $v$ can either refer to 1, 2, or 4 macroblocks, independent of whether integer or sub-pel motion vector precision is used. $\alpha_{i}(v)$ is the share of the $i$ th macroblock in $v$ 's targeted region and is directly related to the labels of edges in Fig. 1. The function $\alpha$ can be further extended to incorporate the optional weighted prediction feature [33], which is not considered in this paper. The recursive computation can be significantly accelerated by caching values of $\delta_{\text {dep }}(m)$ where $m$ is part of a frame of the reference picture list. The mapping from $\delta_{\mathrm{dep}}$ to $\Psi_{\mathrm{dep}}$ is specified in (4). It can be adjusted with the parameter $\kappa_{\text {dep }}$ which has to be appropriately chosen according to the maximum number of allowed reference frames and the GoP structure used; both are already available at encoding time:

$$
\Psi_{\mathrm{dep}}=1-\left(\frac{1}{\delta_{\mathrm{dep}}(m)+1}\right)^{\kappa_{\mathrm{dep}}} \in[0 ; 1] .
$$




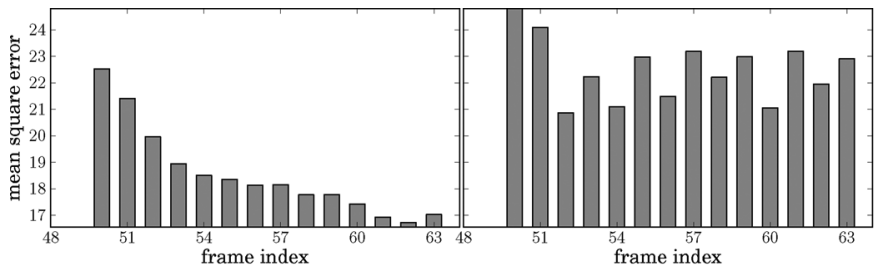

(a)

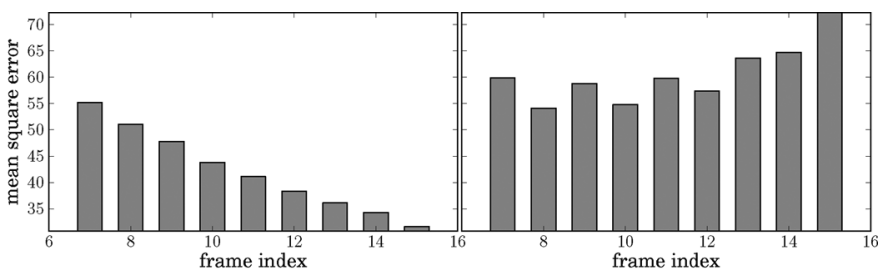

(b)

Fig. 2. Error attenuation when using different encoding settings: bframes $=0$, ref $=1$ (left sub-plots) and $b f$ rames $=1$, re $f=4$. (right sub-plots). (a) Sequence COASTGUARD, slice 2 of frame 50 lost. (b) Sequence STEFAN, slice 3 of frame 7 lost.

It has to be noted that $\Psi_{\mathrm{dep}}$ pessimistically models macroblock distortion by assuming successful reception of all dependency tree leaves. For a discussion on how to incorporate loss-feedback for estimate refinement, the reader is referred to Section IV.

\section{Design of $\Psi_{\mathrm{mv}}$}

When a media unit gets lost, the decoder has to apply interpolation techniques to reduce the resulting distortion in affected and dependent regions. Most error concealment mechanisms use the motion information of neighboring macroblocks to identify suitable regions in temporally collocated frames to aid in reconstructing the lost information. The further apart in any dimension the initially referenced region is, the more unlikely it is that a decoder exploits it for concealment. $\Psi_{\mathrm{mv}}$ considers the length of motion vectors in both spatial and temporal domains, thus incorporating this aspect. Its specification in (5) involves the spatial lengths of motion vectors $v$ given as $\operatorname{len}(v)=\sqrt{v_{x}^{2}+v_{y}^{2}}$ and their temporal extent $\Delta_{\text {ref }}(v)$, which is the difference between the picture timestamps of the current frame and reference frame:

$$
\Psi_{\mathrm{mv}}(m)=\sum_{v \in \mathrm{MV}(m)} \frac{\kappa_{\mathrm{mv}} \cdot \frac{\operatorname{len}(v)}{\operatorname{len}_{\max }}+\left(1-\kappa_{\mathrm{mv}}\right) \cdot \frac{\left|\Delta_{\mathrm{ref}}(v)\right|}{\Delta_{\mathrm{ref}_{\max }}}}{\#(\mathrm{MV}(m))} .
$$

$\operatorname{len}_{\max }$ and $\Delta_{\text {ref }_{\max }}$ are upper bounds on the spatial and temporal prediction distances, respectively, fixed at encoding time, and $\#(\mathrm{MV}(m))$ is the number of motion vectors associated with macroblock $m$. Finally, $\kappa_{\mathrm{mv}}$ balances the impact of spatial and temporal distance on $\Psi_{\text {mv }}$.

\section{Dependency Awareness and Computational Cost}

We remark that the proposed prioritization mechanism, and especially $\Psi_{\mathrm{dep}}$, closely tracks and analyzes motion within sequences to derive estimates. It models temporal error propagation more accurately than approaches discussed in Section II and does not depend on the validity of the conjecture that distortion exponentially decays over time, as assumed by some other models. As simple counterexample to this assumption, we simulated the loss of a randomly chosen NAL unit that belongs to a $\mathrm{P}$-frame with two different test sequences, using the two GoP structures $\mathrm{IP}^{*}$ and $\mathrm{I}(\mathrm{BP})^{*}$, and measured the resulting distortion in subsequent frames. The results, depicted in Fig. 2, suggest that the assumption holds for the $\mathrm{IP}^{*} \mathrm{GoP}$ structure (left sub-plots). However, in the presence of B-frames and when multiple references are allowed (right sub-plots), this may no longer be true. Hence, we do not think that exponential decay distortion models are applicable to arbitrary encoder settings, as, e.g., noted in [23].

ScMP parses besides higher-level information only macroblock types and associated motion vectors. The computational demanding steps of inverse quantization, inverse integer transformation, error concealment simulation, etc. are not needed. This approach can therefore also be applied in real time to highly compressed, high-resolution content which, in contrast, is not true for pixel-level approaches such as $R O P E$ and $A b S$. Hence, it has a wider operational range as it can additionally be used in systems where encoded video content is not pre-stored but created on the fly, possibly coupled with strict timing constraints, such as in video conferencing applications, remote control systems, streaming game technologies, and life-critical medical applications (e.g., tele-surgery).

\section{INCORPORATING FEEDBACK AND TIMING ASPECTS}

The previous section described the prioritization scheme $S c M P$ in a generic manner. This section focuses on the specific properties of live streaming systems which support feedback and selective retransmissions. It demonstrates how feedback can be used to adjust priorities of media units according to the loss history and, consequently, reschedule the send queue by considering timing constraints. We already demonstrated an earlier version of such a system in [3]. However, in that previous approach, estimates were statically derived in advance and were not adjusted according to channel feedback, and the prioritization scheme used is only applicable in combination with MPEG-4 ASP.

Before describing how a transmission's state with respect to time, send queue, and network feedback influences packet priorities, we introduce the concept of send timestamps (sts). An sts is a relative time unit which depends on the decoding timestamps $(d t s)$ and picture timestamps ( $p t s)$ of the related frame and its referenced frames. For derivation, the $s t s$ of each frame is initialized by setting it equal to its $p t s$. Then, it is iterated over all frames in increasing pts order and $\operatorname{sts}\left(f_{j}\right)$ is set to $\operatorname{sts}\left(f_{i}\right)$ if $\operatorname{sts}\left(f_{j}\right)>\operatorname{sts}\left(f_{i}\right)$ and the decoding of frame $f_{i}$ (transitively) depends on frame $f_{j}$ 's availability. sts' are used by the sender application to derive send deadlines for single media units and are advantageous in the presence of backward prediction. Fig. 3 exemplarily depicts the derivation procedure, using an MPEG-2-like GoP structure with single reference targets per prediction direction. Although the example is intentionally kept simple for the purpose of illustration, this concept can be applied to arbitrarily complex inter-prediction structures. By using sts 


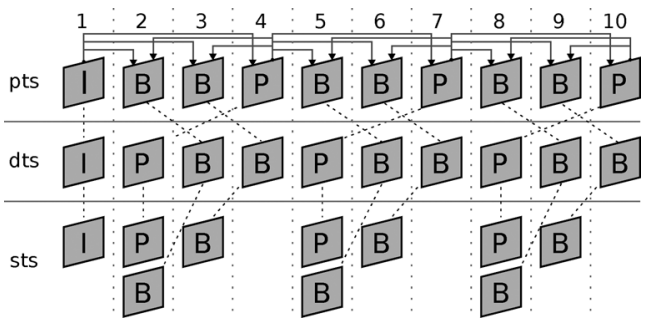

Fig. 3. Mapping of $d t s$ and pts to sts using a simple MPEG-2-like dependency structure. The grey boxes indicate frames that are ordered according to the respective type of timestamp.

over $d t s$ or $p t s$ as primary time unit, the urgency of delivering media units is more transparent to the mechanism which controls the send queue. As depicted in Fig. 3, packets that constitute the B-and $\mathrm{P}$-frames with $s t s=5$ have different values of $p t s$ and $d t s$, although it is of equal urgency (with respect to timeliness) to send either of them because both frames are needed to correctly display the picture with $p t s=5$. Consequently, NAL units of both frames can be sent interchangeably, and further criteria such as $S c M P$ can be taken into account to minimize video quality degradations when the allowed send rate drops below the video bitrate.

To incorporate information about lost units, $S c M P$ has to be modified as follows: recalling $\Psi_{\text {dep }}(m)$, it considers the distortion caused in dependent areas when $m$ gets lost by recursively traversing its inverted dependency graph. This algorithm is modified so that at each recursion step (i.e., at each node $m_{i}$ in the graph), the send queue and the transmission history are inspected to decide whether to skip $m_{i}$ and its sub-branch or not. There are four possible cases with respect to the network packet(s) that contain $m_{i}$ :

1) The packet has been acknowledged. $\Longrightarrow \delta_{\text {dep }}\left(m_{i}\right)$ is evaluated as usual.

2) The packet has not been acknowledged yet, but it has already been emitted. $\Longrightarrow$ Optimistically, it is assumed that the packet will correctly be received, and consequently, $\delta_{\text {dep }}\left(m_{i}\right)$ is evaluated.

3) The packet has not been sent yet or it has timed out, but it will be emitted in time with respect to the current send schedule. $\Longrightarrow$ Similar to case $2, \delta_{\mathrm{dep}}\left(m_{i}\right)$ is evaluated, but its value is reduced according to the proximity of the packet's sts to the sender's notion of "current time".

4) The packet has not been sent yet or it has timed out, and it cannot meet its decoding deadline with regard to the current send schedule. $\Longrightarrow \delta_{\text {dep }}\left(m_{i}\right)=0$.

The idea behind this constrained recursion is to incorporate the co-impact of multiple packet losses. Assume we are in case 4 where $m_{i}$ will not be available at the decoder. Consequently, error concealment mechanisms will have to interpolate missing motion vectors, and unfortunately, the reconstructed vectors quite often differ from the lost originals. Based on that, we consider the additional loss of $m$ to be less severe when we know that some sub-branches of $m$ 's dependency tree are already damaged. As a reason, further distortion of $m_{i}$, and depending regions "inherited" from $m$ by temporal propagation, will in general less drastically (and in some cases negligibly) degrade its video quality.

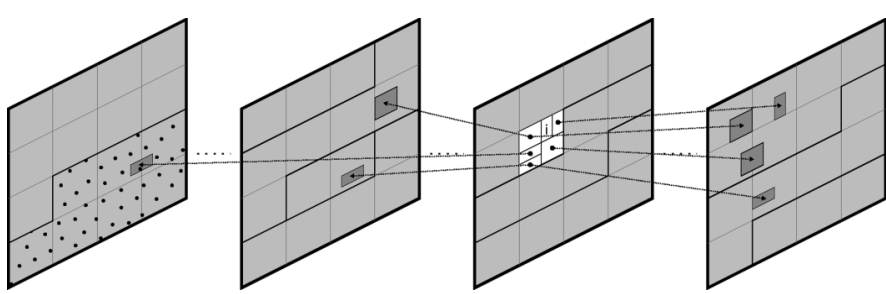

Fig. 4. Motion vectors which point to lost (dotted) slices are ignored by the feedback-adaptive version of $\Psi_{\text {miv }}$.

As discussed in Section III-C, $\Psi_{\mathrm{mv}}(m)$ addresses the locally introduced distortion by the hypothetical loss of $m$ by estimating the chances of error concealment mechanisms to find the parent regions based on the spatio-temporal extents of motion vectors. To incorporate information about loss which affects these regions, the corresponding addends are skipped in the evaluation of (5). As a reason, even if some decoders' error concealment techniques are able to locate the original area used for reference, the quality of the reconstruction would still be less satisfactory due to the amplifying effect of inherited mis-concealments. Fig. 4 depicts a snapshot of a transmission from the sender application's perspective to illustrate the idea behind the previously proposed modification, in which dotted areas represent slices that will not be available at the decoder at their $d t s$ with high probability. Consequently, motion vectors that point from $m$ to such regions will not be considered by $\Psi_{\mathrm{mv}}(m)$.

$\Psi_{\text {type }}(m)$ is left untouched because it does not consider dependencies between media units and can therefore be evaluated independently of the current loss history. In conclusion, previous modifications were designed in a rather intuitive manner, but they positively influence video quality as will be shown in Section V. To distinguish between the original proposal of $S c M P$ described in Section III and the modified, feedback-adaptive version proposed in this section, the terms $S c M P_{\text {fixed }}$ and $S c M P_{\text {adapt }}$ will be used for the remainder of this paper.

Next, we present a simple way to adequately combine the two attributes timeliness and relevance of content $(\Psi)$ as a weighted sum (adjusted by the parameter $w_{\mathcal{P}}$ ). This yields a joint rating based on which the ranking $\mathcal{P}$ of pending packets in the sender's buffer can be determined (6):

$$
\mathcal{P}(p)=w_{\mathcal{P}} \cdot \Psi(p)+\left(1-w_{\mathcal{P}}\right) \cdot \frac{\operatorname{now}()-t_{S}-\frac{\operatorname{sts}(p)}{f p s}}{t_{D}-t_{S}} .
$$

The idea behind $\mathcal{P}$ 's design is, as in [27], to preferentially send packets which get precariously close to their sending deadline, i.e., when now () is close to $t_{D}+\operatorname{sts}(p) / f p s$. The transmission of packets whose temporal margin is comparatively large (when now () is close to $\left.t_{S}+\operatorname{sts}(p) / f p s\right)$ should be deferred.

To explain these variables, we first have to make some assumptions that concern the minimalistic streaming system used: upon request, the sender application immediately (at time $t_{S}$ ) starts transmitting/forwarding a stream obtained from an arbitrary source over an error-prone channel to the receiver at the highest possible rate. The size of the send queue may fluctuate depending on the percentage of intra-coded macroblocks in a frame and the extent of packet loss experienced along the path 
to the receiver. The data arrival rate at the sender is assumed to be fairly constant on a per-GoP basis and packets are ordered according to their payloads' position in the bitstream, i.e., the function $\Psi$ is constant throughout the initial buffering phase. Each packet which arrives at the receiver is immediately acknowledged. The feedback channel is assumed to be error-free which can be realized, e.g., by applying strong FEC. The receiver starts displaying the video after having received at least $\beta$ frames, where this number may be communicated to the sender or may be fixed in advance [e.g., by considering supplemental enhancement information (SEI) recovery points in the stream]. In this connection, we define $t_{D}$ as the time when the sender emits the last packet of the stream's first $\beta$ frames (commonly referred to as start-up delay). This is used together with the stream's frame rate fps and the current time now() to convert relative sts of packets to absolute send deadlines. The terms $t_{D}-t_{S}+F T T$ and $\beta / f p s$ with $F T T$ being the forward trip time can be regarded as the overall delay introduced by the streaming system and its lower bound, respectively. The magnitude of $t_{D}$ determines the system's elasticity and its ability to compensate packet loss and video bitrate variation. The choice of $\beta$, which directly influences $t_{D}$, is a tradeoff between robustness and delay.

For the sake of completeness, we point out that the decoder in our setup strictly adheres to sts of media units and ignores packets which arrive after this deadline. It would, however, theoretically be possible to implement multiple decoding loops upon the arrival of delayed NAL units to decrease the distortion in dependent, non-decoded frames, which would stop the error propagation caused by delayed packets [34]. Furthermore, we note that the send mechanism used is non-adaptive with respect to network delay fluctuations and that the display offset $t_{D}-t_{S}$ is assumed to be fairly constant throughout an entire streaming session. This might be an unrealistic assumption in some distribution networks. Yet, it is not the goal of this work to develop sophisticated mechanisms that closely track network delay, but to evaluate streaming systems that use different content-aware prioritization schemes, which can still be realistically conducted facing this restriction. An alternative would have been to derive the probability for each packet to reach the sender on time, based on the history of recently tracked $F T T$ s, and to appropriately inject these probabilities into (6), which would, however, render the approach unnecessarily complicated.

\section{Performance Evaluation}

\section{A. Reference Approaches}

As reference approaches, we selected $A b S$ and $S T p$, discussed in Section II. As a reminder: $A b S$ simulates the loss of single media units and calculates the resulting distortion at the pixel level, whereas $S T p$ uses the types of slices as decision criterion. Thereby, STp considers intra-coded slices as most important and inter-coded slices of $\mathrm{P}$-frames to be medium important whereas inter-coded bidirectional predicted slices of B-frames are of low relevance. We justify the choice of the reference mechanisms as follows: $A b S$ is a popular, frequently cited approach, there are several remarkable parallels to other papers, and the algorithm is well-specified, i.e., the risk of introducing flaws in the imple-
TABLE I

Static Parameters Used in the Performance Evaluation

\begin{tabular}{|c|c|c|c|}
\hline Category & Parameter & \multicolumn{2}{|c|}{ Value } \\
\hline \multirow{8}{*}{ Encoder } & GoP structure & $\mathrm{IbBb}(\mathrm{PbBb})^{3}$ & $\overline{\mathrm{I}(\mathrm{bP})^{*}}$ \\
\hline & keyint & \multicolumn{2}{|c|}{$\frac{1}{16}$} \\
\hline & bframes & 3 & 1 \\
\hline & $r e f$ & 4 & 1 \\
\hline & intra-refresh & no & yes \\
\hline & fps & \multicolumn{2}{|c|}{30} \\
\hline & $l e n_{\max }$ & \multicolumn{2}{|c|}{256} \\
\hline & $\Delta_{\text {ref }_{\max }}$ & \multicolumn{2}{|c|}{16} \\
\hline \multirow{2}{*}{ Streamer } & $w_{\mathcal{P}}$ & \multicolumn{2}{|c|}{0.5} \\
\hline & $\beta$ & 10 & 6 \\
\hline \multirow{5}{*}{$\Psi_{\mathrm{ScMP}_{*}}$} & $w_{\text {type }}$ & \multicolumn{2}{|c|}{0.27} \\
\hline & $w_{\text {dep }}$ & \multicolumn{2}{|c|}{0.44} \\
\hline & $\kappa_{\text {dep }}$ & \multicolumn{2}{|c|}{0.4} \\
\hline & $w_{\mathrm{mv}}$ & \multicolumn{2}{|c|}{0.29} \\
\hline & $\kappa_{\mathrm{mV}}$ & \multicolumn{2}{|c|}{0.652} \\
\hline
\end{tabular}

mentation is low. Similarly, $S T p$ is repeatedly used in scientific experiments to bring in content-awareness at which mostly one slice per picture is assumed. The procedure is easy to implement especially on a per-frame basis, and it serves in this context as representative of the class of intuitive, lightweight approaches.

As already pointed out in Section II, the computations needed by $A b S$ are hard to conduct in real time. To evaluate the performance of $A b S$, necessary calculations were therefore carried out in advance, and the results were fed into the sender's scheduler from a cache. The priorities were not derived using a model-based approach as proposed, e.g., in [26], but exhaustively simulated by entirely decoding the sequence for each NAL unit. Consequently, these values are, although obtained at an unreasonable computational cost, of higher precision than estimates delivered by model-based techniques. They can even be considered as an upper bound in terms of prioritization precision in situations where packet loss only occurs isolated.

The send mechanism enqueues packets according to the ranking specified in (6), in which the relevance of content is determined by one of the distortion estimation schemes $\Psi_{\mathrm{ScMP}_{\text {fixed }}}, \Psi_{\mathrm{ScMP}_{\text {adapt }}}, \Psi_{\mathrm{AbS}}$, and $\Psi_{\mathrm{STp}}$. Regarding the weighting parameter, $w_{\mathcal{P}}=1 / 2$ turned out to be a reasonable value. For the sake of completeness, we also considered the performance of a content-unaware system using sts-based earliest-first $(E F)$ scheduling. The corresponding function $\mathcal{P}_{\mathrm{EF}}$ in (6) can be obtained by fixing $\Psi$ or setting $w_{\mathcal{P}}=0$.

We focused on two GoP structures which exploit the benefit of bidirectional prediction to different degrees. They are listed in Table I together with all relevant system parameters, and their choice will be justified in Sections V-B and V-E. B-frames indicate referencable frames whereas b-frames cannot be used as reference for temporal prediction. The GoP structures are completely defined by the encoder parameters keyint and $b$ frames, which are the key-frame interval and the maximum possible number of consecutive B-/b-frames, respectively. Depending on the content, the encoder may adaptively decide to skip certain B-/b-frames, which leads to irregular GoP structures. ref is the maximum length of forward/backward prediction lists and intra-refresh instructs the encoder to distribute intra-coded macroblocks evenly across multiple frames, a technique whose benefit will be explained in Section V-E. Suitable values for the 


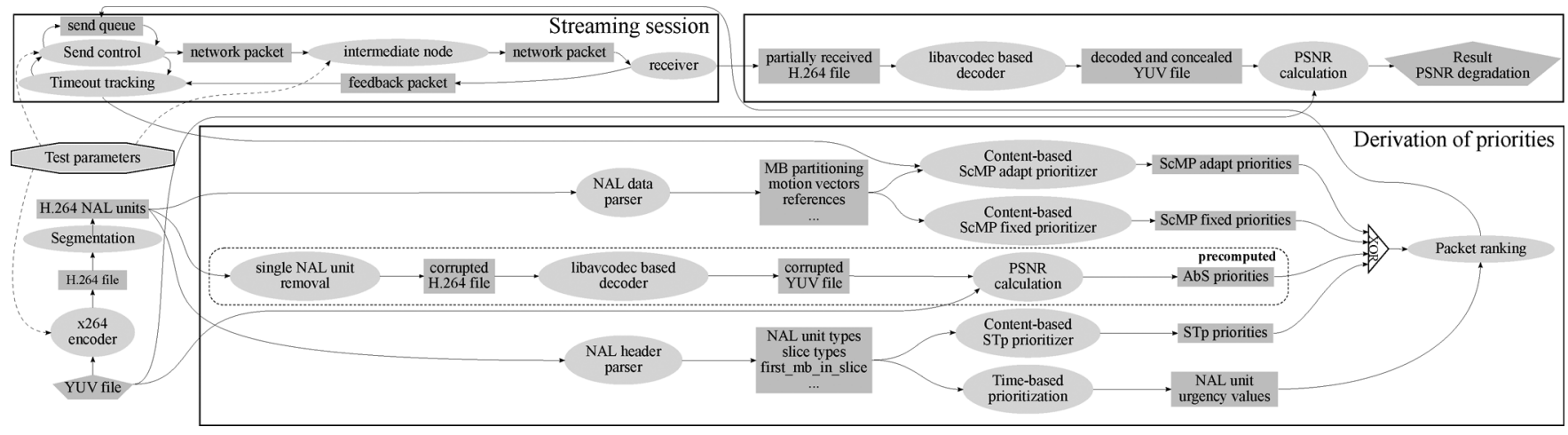

Fig. 5. Simulation setup: Inputs are the YUV file of a selected test sequence and a set of test parameters determining encoding settings and transport-specific settings like bandwidth, $R T T, R T O, \beta$, etc. The output is the derived video quality degradation, obtained by using a specific NAL unit prioritization technique. The building block "Streaming session" is both realized as simulation using $d f$ Sim and as experiment in a real wired/wireless network as described in Section V-B.

parameters related to $\Psi_{\mathrm{ScMP}_{*}}$ were determined in offline-simulations and are independent of the target streaming system and content protection mechanism.

\section{B. Simulation Setup}

For encoding all test sequences, we used an up-to-date version of the open source H.264/MPEG-4 AVC encoder x264, presently being the best performing H.264 encoder software implementation according to [35]. All test sequences considered in experiments are commonly used in scientific literature and are publicly available. ${ }^{3}$ We adjusted the maximum size of slices according to the network's maximum transfer unit, used a (rather error-robust) closed-GOP size of 16 frames, directed the encoder to produce a stream at a roughly constant bitrate, and limited the size of prediction lists to 4 frames (cf. parameter ref in Table I). Because we primarily consider live streams, the encoder is only allowed to introduce a small amount of latency. When streaming a live event such as a football game over a content distribution network, delay is not that critical since the consumer is not interacting with the content she/he is viewing and a time-shift of several seconds may be tolerable. However, interactive use cases like video conferencing become completely unusable when latency is in the range of seconds. We therefore limited all look-ahead features such as macroblock-tree rate control and only allowed at most 3 consecutive bi-directionally encoded frames $(\mathrm{bBb})$ to keep the latency of the stream below $200 \mathrm{~ms}$ at a rate of 30 frames/s. To further reduce delay and encode a video with the smallest amount of latency possible, all lookaheads would have to be turned off and B-/b-frames should not be used at all, but with the consequence that the compression ratio further decreases. Thus, our configuration can be regarded as an acceptable compromise between latency and compression efficiency in interactive streaming scenarios.

For decoding and concealment, we used the latest version of the open source video coding library libavcodec,${ }^{4}$ which supports motion vector copy error concealment and applies strong deblocking filtering to damaged macroblocks. The decrease in video quality caused by transmission errors is expressed

${ }^{3}$ YUV test sequences taken from http://media.xiph.org/video/derf/

${ }^{4}$ libavcodec ver. 0.6.2 of FFmpeg, http://www.ffmpeg.org as decrement in peak signal-to-noise ratio (PSNR), which is easier to interpret than mean-squared errors (MSE), since the relationship between perceived subjective quality and PSNR is considered to be roughly linear when values are in the range of 20 to 40 [36]. All presented PSNR values in this paper cover both channel induced distortion as well as encoding distortion and range from $36 \mathrm{~dB}$ to $41 \mathrm{~dB}$ when no channel errors occur, thus ensuring that averaging over frame PSNRs does not lead to skewed results. Due to all test sequences used being rather short (250-1000 frames each), the average over all frame-PSNRs can be regarded as a reasonable video quality indicator.

The performance of all five prioritization techniques was evaluated in three different experimental environments:

- in a wired testbed where loss and delay are primarily introduced at an intermediate node;

- in a transmission simulator where loss and delay models cause artificial data loss;

- and in a wireless environment where packet loss can be mainly attributed to signal interferences.

The two former setups, used for performance evaluations in Sections V-C-V-E, are explained in this section, whereas the discussion of the wireless setup is deferred to Section V-F. The wired testbed consists of a sending node, a receiving node, and an intermediate node which is responsible for adjusting connection settings like delay, bandwidth, and loss via the Linux kernel module netem [37]. A sketch of the entire simulation setup is depicted in Fig. 5. For all experiments, we used UDP as transmission protocol and set the available link bandwidth based on the average bitrate of the encoded sequence plus an additional overhead of $5 \%-10 \%$ to be used for compensating bitrate fluctuations and enabling a limited number of retransmissions.

In contrast to the wired setup, we initially used our software dfSim, a transmission simulator to simulate unreliable unicast video streams, to conduct quick offline experiments. dfSim supports various loss and delay models, it enables the exact reproducibility of outcomes of experiments by using equal seeds, and it provides convenient means for parameter tuning. Furthermore, it has advanced connection tracking and analysis capabilities, and it significantly speeds up batch processing. After initial test runs, it turned out that the results obtained by using $d f$ Sim are highly similar to those coming from the wired testbed. Because of this, and because of the fact that obtaining a sufficiently 


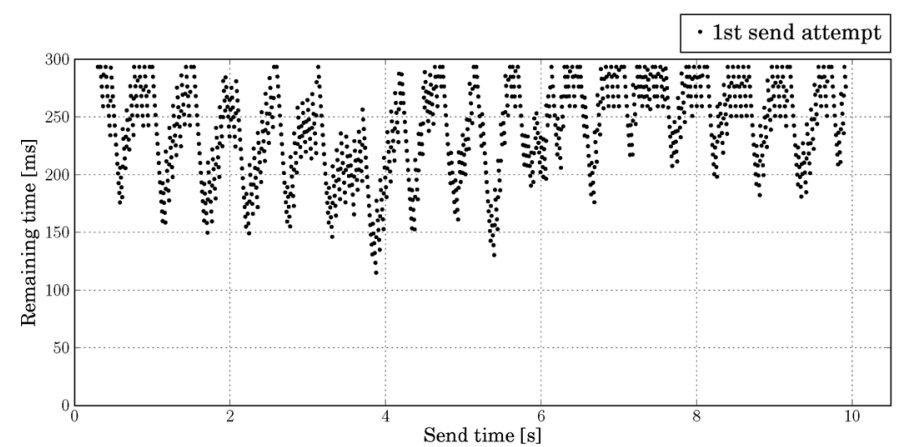

(a)

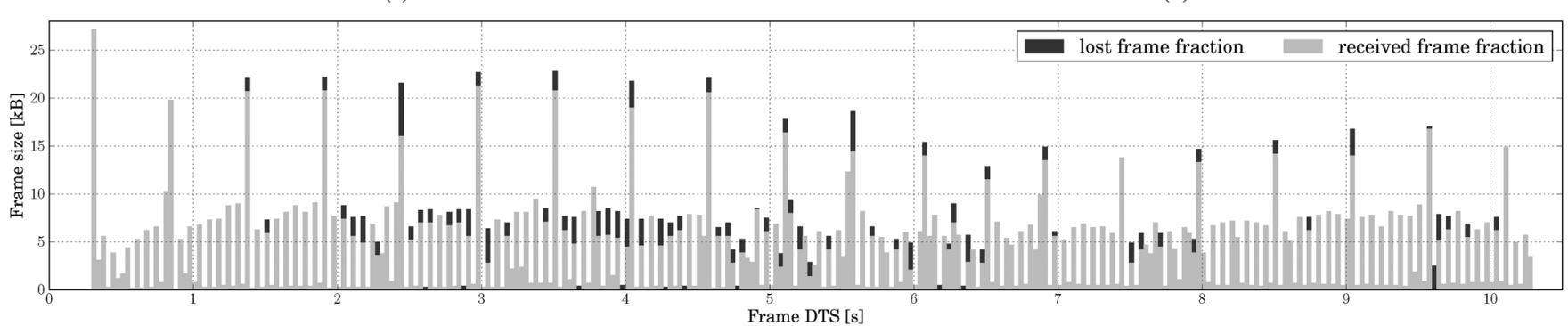

(c)

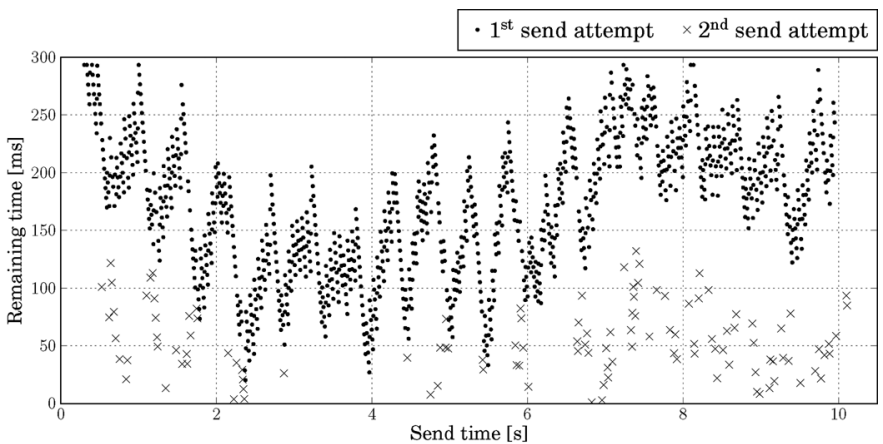

(b)

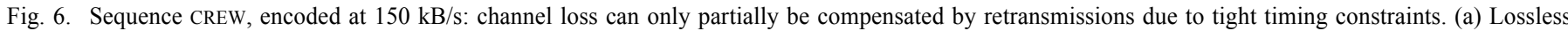
communication channel. (b) $16 \%$ Bernoulli loss. (c) Impact of channel loss in (b) on the availability of frames at the receiver.

large number of results $(n \geq 100)$ per test sequence to derive expressive mean values would have been a very time-consuming process when merely conducted in the wired testbed, approximately $75 \%$ of the derived samples in Sections V-C-V-E originate from simulations done with $d f$ Sim. As a positive side effect, common seeds (per run) could be used for the loss/delay generators, which enabled the comparison of different prioritization under exactly the same network conditions, thus improving the comparability amongst results.

For simplicity, we assume that the duration between a packet arriving at the receiver and the receiver acknowledging the packet is negligible and that feedback is not affected by loss. The parameter $\beta$, which influences the system's overall delay as explained in Section IV, is set to 10 frames. By using a round-trip time $(R T T)$ of $120 \mathrm{~ms}$ and a rather tight retransmission timeout $(R T O)$ of $1.2 \cdot R T T$, this means that it is frequently possible to make at least one retransmission attempt. In rare situations, even a second retransmission might be feasible. The appropriate choice of $\beta$ depends on the delay of the network path, the steadiness of the video bitrate, and the constraints of the application scenario. With regard to the $R T O$, we underline that this paper primarily focuses on the performance evaluation of loss-impact prediction techniques. Consequently, the occurrence of large delay fluctuations is excluded by setting the delay's standard deviation parameter within $d f$ Sim/netem to a low value $(\sigma=5 \mathrm{~ms})$. This keeps the percentage of spurious timeouts close to zero. The decision to fix the $R T O$ at $1.2 \cdot R T T$ was made for the sake of simplicity by considering the way TCP predicts retransmission timeouts: According to [38], the $R T O$ is estimated by $S R T T+\max (G, 4 \cdot R T T V A R)$ where $S R T T$ is an exponentially-weighted moving average (EWMA) of the past RTT samples, $G$ is the kernel's clock granularity (in our case $100 \mathrm{~Hz}$ ), and RTTVAR is the smoothed RTT standard deviation estimator, also derived as an EWMA. By inserting the corresponding values, we get $120 \mathrm{~ms}+\max (10 \mathrm{~ms}, 4 \cdot 5 \mathrm{~ms})=140 \mathrm{~ms}$ which is notably close to $144 \mathrm{~ms}$, the value we used. We deliberately neglect the constraint $R T O \geq 1 \mathrm{~s}$ demanded in [38] which is naturally too costly for delay-sensitive video applications, and ignore the exponential timer backoff.

Although these settings are compliant with the experiments' setup and sufficiently satisfy our needs, we emphasize that an accurate $R T O$ predictor forms the basis of any retransmission scheme to be applicable to environments that suffer from large delay fluctuations. The $R T O$, when derived at the application level, should ideally be adapted to the relevance of content and the playout buffer state of the streaming system, as proposed in [39].

To demonstrate the impact of tight timing constraints by example, we selected an arbitrary instance of a test series, depicted in Fig. 6. Each NAL unit, once available at the sender, has a certain lifetime in the queue during which it has to be transmitted to be displayed at the receiver on time. If no loss occurs [Fig. 6(a)], all packets can be sent on time, whereas if there is a considerable amount of loss [Fig. 6(b)], some packets exceed their life span while waiting to be sent or get lost during transit, and their retransmission timer expires too late to allow a second transmission attempt. As a result, parts of frames are not available at the receiver at decoding time [Fig. 6(c)], and the decoder has to conceal corresponding regions. Clearly, content-aware selective retransmission schemes are a proper means to decrease the degradation in video quality in such situations.

\section{Streaming Under Independent Loss}

In a first test series, we generated loss events using the Bernoulli model at loss rates of up to $25 \%$. All obtained results 


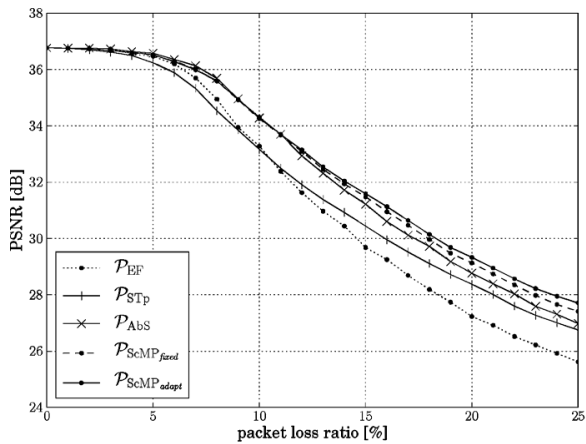

(a)

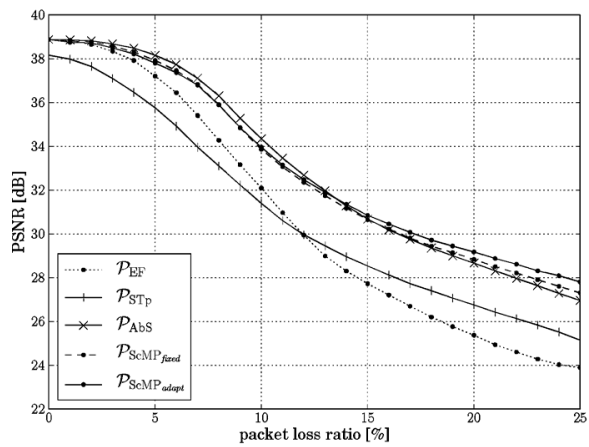

(d)

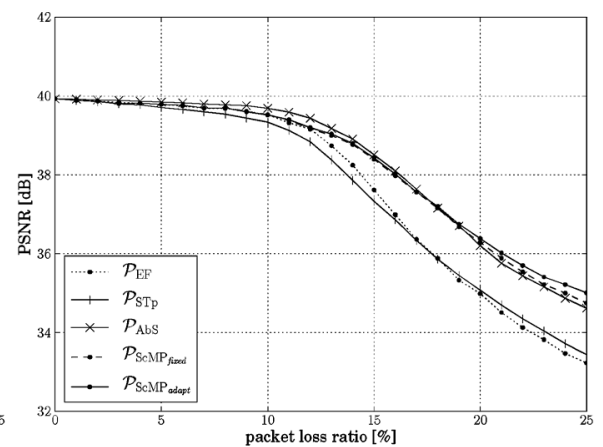

(b)

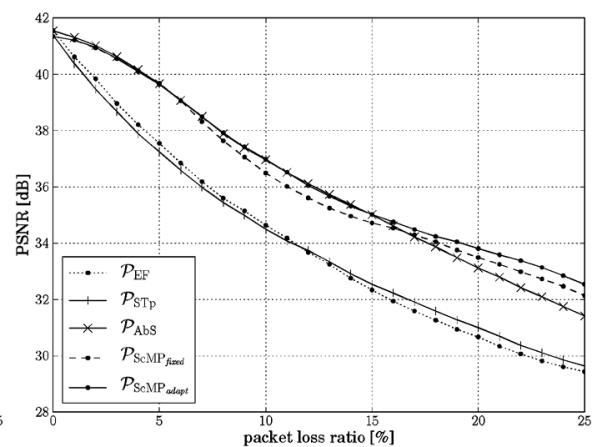

(e)

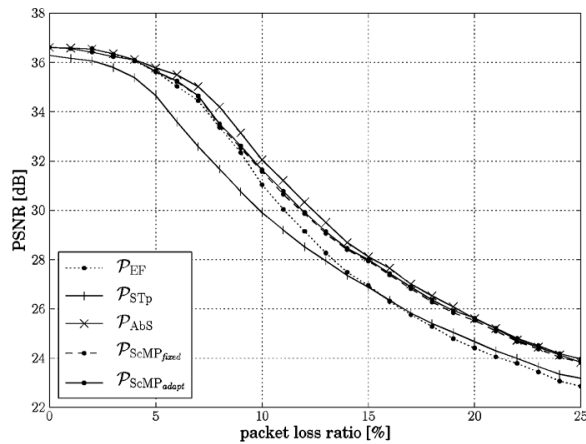

(c)

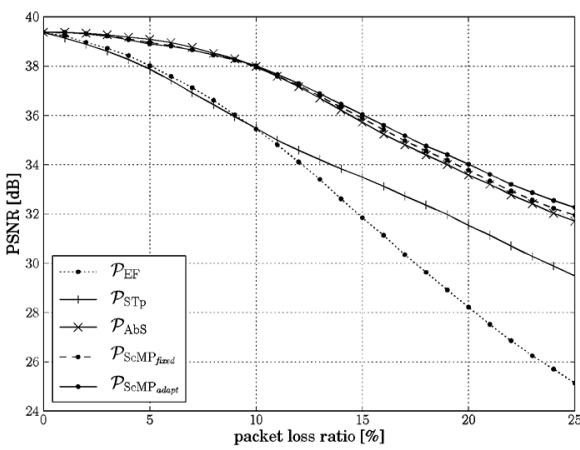

(f)

Fig. 7. Performance of prioritizations under independent loss; parameters: bframes $=3$, ref $=4, \beta=10$. (a)-(c) are low-resolution CIF sequences and (d)-(f) are high-resolution $720 \mathrm{p}$ sequences. Recall that $\Psi_{\mathrm{EF}}, \Psi_{\mathrm{STp}}, \Psi_{\mathrm{ScMP}}$ fixed $_{\text {f }}$, and $\Psi_{\mathrm{ScMP}}$ arap real-time capable and can be derived on the fly whereas $\Psi_{\mathrm{AbS}}$ depends on heavy precomputation, thus making it inapplicable to live streaming applications. Additionally, $\Psi_{\mathrm{AbS}}$ profits from the use of PSNR as video quality metric as will be discussed in Section V-G. (a) COASTGUARD. (b) CONTAINER. (c) MOBILE. (d) MOBCAL. (e) PARKRUN. (f) WATERFALL.

TABLE II

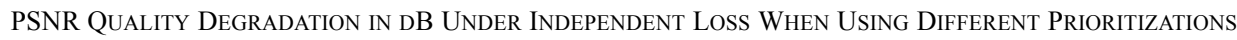

\begin{tabular}{|c|c|c|c|c|c|c|c|c|c|c|c|c|c|c|c|c|}
\hline \multirow{2}{*}{\multicolumn{2}{|c|}{$\begin{array}{c}\text { Prioritization } \\
\text { Loss rate }\end{array}$}} & \multicolumn{3}{|c|}{$\mathrm{EF}$} & \multicolumn{3}{|c|}{ STp } & \multicolumn{3}{|c|}{$\mathrm{AbS}$} & \multicolumn{3}{|c|}{$\mathrm{ScMP}_{\text {fixed }}$} & \multicolumn{3}{|c|}{$\mathrm{ScMP}_{\text {adapt }}$} \\
\hline & & $8 \%$ & $16 \%$ & $24 \%$ & $8 \%$ & $16 \%$ & $24 \%$ & $8 \%$ & $16 \%$ & $24 \%$ & $8 \%$ & $16 \%$ & $24 \%$ & $8 \%$ & $16 \%$ & 2 \\
\hline Name & Resolutio & & & & & & & & & & & & & & & \\
\hline CITY & & 7.17 & 32.07 & 8.34 & 6.57 & 1.95 & 28.75 & 37.98 & 33.37 & 29.81 & 7.80 & 33.39 & 30.19 & 37.81 & 33.45 & 30.3 \\
\hline DASTGUARD & 88 & 4.95 & 29.25 & 5.92 & 4.50 & 0.00 & 27.01 & 35.69 & 30.60 & 27.38 & 35.59 & 30.95 & 27.66 & 35.60 & 31.13 & 27.95 \\
\hline ONTAINER & & 9.69 & 36.99 & 3.47 & 9.54 & 36.86 & 33.72 & 39.78 & 38.10 & 34.87 & 39.70 & 37.98 & 35.00 & 39.70 & 38.02 & 35.22 \\
\hline REW & & 1.72 & 9.92 & 7.05 & 1.24 & 0.30 & 7.83 & 35.08 & 0.56 & 27.77 & 5.87 & 31.79 & 8.76 & 5.89 & 31.92 & 28.99 \\
\hline FOOTBALL & & .74 & 24.83 & 22.53 & 3.57 & 1 & 3.31 & 9.68 & 25.85 & 23. & 9.52 & 25.87 & 23.57 & 9.52 & 25.92 & 23.71 \\
\hline FOREMAN & & 4.74 & 26.69 & 24.08 & 34.63 & 28.29 & 26.02 & 37.34 & 31.34 & 27.55 & 37.30 & 31.55 & 27.92 & 37.44 & 31.76 & 28.18 \\
\hline BOUR & & 3.28 & 29.02 & 26.50 & 33.19 & 29.92 & 27.62 & 33.58 & 29.58 & 27.02 & 33.52 & 30.24 & 27.94 & 33.56 & 30.44 & 28.42 \\
\hline SHWAY & & 2.23 & 29.45 & 27.44 & 33.63 & 31.21 & 29.24 & 34.58 & 31.63 & 29.45 & 34.32 & 31.39 & 29.35 & 34.49 & 31.58 & 29.51 \\
\hline ICE & & 5.29 & 27.27 & 24.27 & 34.61 & 28.37 & 25.59 & 37.61 & 29.88 & 26.50 & 37.22 & 29.80 & 26.76 & 37.36 & 29.97 & 26.96 \\
\hline MOBCAL & $280 \times 720$ & 4.28 & 27.21 & 24.03 & 33.10 & 28.13 & 25.52 & 36.31 & 30.19 & 27.29 & 35.91 & 30.23 & 27.61 & 35.90 & 30.46 & 28.09 \\
\hline MOBILE & 288 & 3.37 & 26.32 & 23.07 & 31.69 & 26.36 & 23.36 & 34.20 & 27.65 & 24.14 & 33.56 & 27.38 & 24.04 & 33.51 & 27.44 & 24.18 \\
\hline NEWS & 38 & .14 & 31.31 & 28.37 & 35.05 & 31.40 & 28.88 & 38.88 & 36.30 & 33.55 & 38.21 & 36.05 & 33.46 & 38.47 & 36.19 & 33.50 \\
\hline RKRUN & 20 & 60 & 31.94 & 29.61 & 35.45 & 32.23 & 29.86 & 37.89 & 34.62 & 31.75 & 37.64 & 34.54 & 32.47 & 37.92 & 34.76 & 32.85 \\
\hline MPETE & & & 30.06 & 26.71 & .61 & 30.16 & 27.11 & 4.07 & 30.93 & 27.59 & 34.01 & 30.90 & 27.66 & 34.03 & 30.99 & 27.87 \\
\hline WATERFALL & $880 \times 720$ & 36.62 & 31.14 & 25.71 & 36.44 & 33.13 & 29.89 & 38.53 & 35.24 & 32.03 & 38.45 & 35.42 & 32.23 & 38.69 & 35.50 & 32.49 \\
\hline
\end{tabular}

are summarized in Table II at three different loss rates, and selected sequences are depicted in Fig. 7. It can be observed that $\mathcal{P}_{\mathrm{ScMP}_{\text {fixed }}}$ performs quite similar to $\mathcal{P}_{\mathrm{AbS}}$. More specifically, $\mathcal{P}_{\text {AbS }}$ provides slightly better results at low loss rates whereas $\mathcal{P}_{\mathrm{ScMP}_{\text {fixed }}}$ tends to outperform $\mathcal{P}_{\mathrm{AbS}}$ at higher loss rates. A possible explanation is that $\Psi_{\mathrm{AbS}}$ is based on the impact of independently lost NAL units whereas the design of $\Psi_{\mathrm{ScMP}_{\text {fixed }}}$ incorporates dependencies between NAL units. Consequently, at high loss rates when the probability of NAL units being independently lost is lower, $\Psi_{\mathrm{ScMP}_{\text {fixed }}}$ provides more accurate results than $\Psi_{\mathrm{AbS}}$. Compared to that, $\mathcal{P}_{\mathrm{ScMP}_{\text {adapt }}}$ provides similar results as $\mathcal{P}_{\mathrm{ScMP}_{\text {fixed }}}$, but it shows an improved performance at high loss rates due to the incorporation of network feedback (cf. Fig. 5). As expected, the use of each of the three sophisticated prioritization mechanisms leads to a considerable increase in video quality when compared to the content-unaware prioritization $\mathcal{P}_{\mathrm{EF}}$. Surprisingly, the results of $\mathcal{P}_{\mathrm{ST}}$ were below expectations. In some cases, it performed even worse than $\mathcal{P}_{\mathrm{EF}}$, which indicates that merely considering the types of slices as importance indicator, although easy to implement, is not sufficient. 


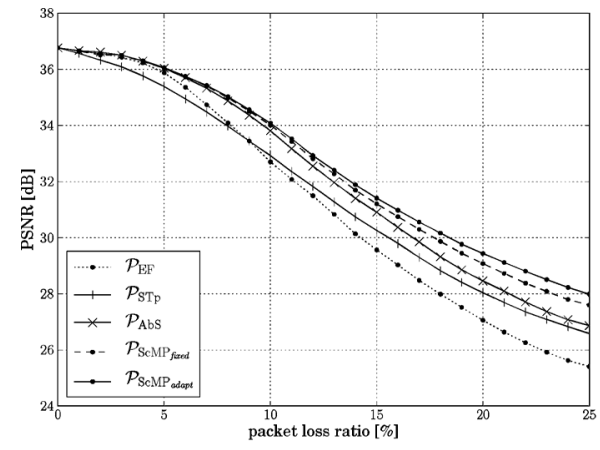

(a)

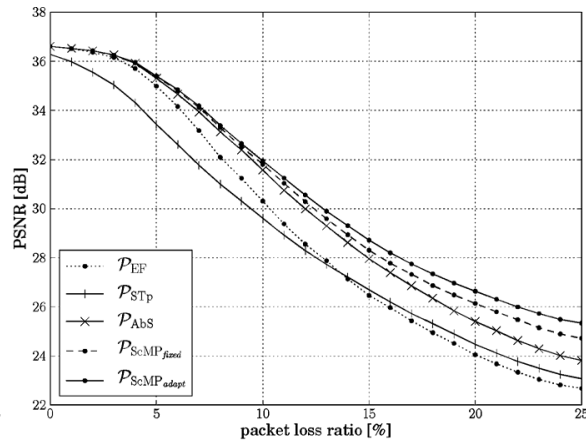

(b)

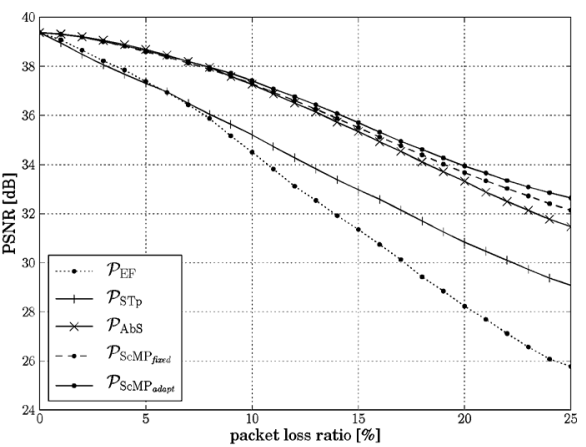

(c)

Fig. 8. Performance of prioritizations under bursty loss; parameters: b f r ames $=3$, ref $f=4, \beta=10$. (a) COASTGUARD. (b) MOBILE. (c) WATERFALL.

TABLE III

PSNR QUALITY DEGRADATION IN DB UNDER BURSTY LOSS WHEN USING DIFFERENT PRIORITIZATIONS

\begin{tabular}{|c|c|c|c|c|c|c|c|c|c|c|c|c|c|c|c|c|}
\hline \multirow{2}{*}{\multicolumn{2}{|c|}{$\begin{array}{c}\text { Prioritization } \\
\text { Loss rate }\end{array}$}} & \multicolumn{3}{|c|}{$\mathrm{EF}$} & \multicolumn{3}{|c|}{ STp } & \multicolumn{3}{|c|}{$\mathrm{AbS}$} & \multicolumn{3}{|c|}{$\mathrm{ScMP}_{\text {fixed }}$} & \multicolumn{3}{|c|}{$\mathrm{ScMP}_{\text {adapt }}$} \\
\hline & & $8 \%$ & $16 \%$ & $24 \%$ & $8 \%$ & $16 \%$ & $24 \%$ & $8 \%$ & $16 \%$ & $24 \%$ & $8 \%$ & $16 \%$ & $24 \%$ & $8 \%$ & $16 \%$ & 2 \\
\hline Name & Resol & & & & & & & & & & & & & & & \\
\hline CITY & & .04 & 1.60 & 7.95 & 5.29 & 1.44 & 8.50 & 6.95 & 32.85 & 9.54 & 6.99 & 33.15 & 30.03 & 77.08 & 33.41 & 30.49 \\
\hline ASTGUARD & 288 & 4.09 & 29.03 & 25.63 & 3.97 & 9.80 & 26.83 & 4.88 & 30.37 & 27.08 & 34.99 & 30.75 & 27.80 & 5.03 & 30.98 & $\overline{28.23}$ \\
\hline ONTAINER & 8 & 7.48 & 34.93 & 2.17 & 7.18 & 35.09 & 2.63 & 8.01 & 35.97 & 33.76 & 8.04 & 36.15 & 33.99 & 8.02 & 36.25 & 34.21 \\
\hline $\mathrm{EW}$ & & .45 & 9.32 & 6.56 & 3.81 & 30.14 & 7.62 & 3.96 & 29.98 & 27.27 & 4.99 & 31.40 & 28.32 & 5.02 & 1.58 & 28.57 \\
\hline FOOTBALL & & 35 & 24.61 & 22.39 & 8.41 & 25. & 23.26 & 9.67 & 25.91 & 23.3 & 28.52 & 25.99 & 23.52 & 28.53 & 26.05 & 23.61 \\
\hline EMAN & & 2.09 & 26.56 & 23.99 & 32.42 & 27.99 & 25.51 & 35.30 & 30.53 & 27.00 & 35.25 & 30.60 & 27.12 & 35.25 & 30.72 & 27.45 \\
\hline HARBOUR & 76 & 2.77 & 28.82 & 26.22 & 32.89 & 29.86 & 27.43 & 33.15 & 29.44 & 26.77 & 33.10 & 30.10 & 27.76 & 33.25 & 30.39 & 28.15 \\
\hline HIGHWAY & & 1.84 & 29.01 & 26.86 & 33.21 & 30.76 & 28.62 & 34.11 & 31.25 & 28.90 & 33.87 & 31.04 & 28.79 & 33.95 & 31.16 & 28.91 \\
\hline ICE & 76 & 5.31 & 26.89 & 24.02 & 34.40 & 28.34 & 25.36 & 37.10 & 29.66 & 26.37 & 36.92 & 29.63 & 26.25 & 37.02 & 29.75 & 26.71 \\
\hline MOBCAL & $80 \times 720$ & 34.05 & 26.83 & 22.95 & 33.44 & 27.11 & 24.65 & 36.12 & 28.56 & 26.23 & 35.85 & 28.45 & 26.21 & 35.89 & 28.49 & 26.40 \\
\hline MOBILE & $52 \times 288$ & 32.10 & 25.97 & 22.82 & 31.00 & 26.20 & 23.25 & 33.13 & 27.40 & 24.02 & 33.30 & 27.78 & 24.90 & 33.39 & 28.20 & 25.49 \\
\hline NEWS & 8 & .92 & 32.27 & 29.03 & 4.95 & 32.12 & 29.45 & 38.13 & 35.19 & 31.89 & 38.18 & 35.99 & 33.30 & 38.18 & 36.14 & 33.45 \\
\hline RKRUN & 0 & 38 & 1.22 & 28.89 & .30 & 31.42 & 29.23 & 36.89 & 33.67 & 30.87 & 36.71 & 33.60 & 30.99 & 36.74 & 33.66 & 31.09 \\
\hline TEMPETE & & 03 & 29.49 & 26.28 & 32.60 & 29.64 & 26.78 & 33.38 & 30.40 & 27.26 & 32.80 & 29.97 & 27.28 & 32.85 & 30.11 & 27.42 \\
\hline WATERFALL & $80 \times 720$ & 35.88 & 30.75 & 26.08 & 36.05 & 32.59 & 29.38 & 37.96 & 34.93 & 31.77 & 37.88 & 35.12 & 32.41 & 37.96 & 35.32 & 32.86 \\
\hline
\end{tabular}

\section{Streaming Under Bursty Loss}

Most related work assumes loss to only occur isolated. However, most studies about network loss behavior agree that packet loss in best-effort networks often exhibits finite temporal dependencies [40], [41], which is especially true for wireless and mobile networks [42]. Consequently, in a second test series, we used Markov models triggering bursty loss behavior to further investigate whether the prioritization techniques proposed in this paper indeed perform better than other approaches. More specifically, we used the Gilbert-Elliot model $\operatorname{GE}\left(p_{G \rightarrow B}, p_{G \leftarrow B}, p_{G}, p_{B}\right)$ [43], [44] where $p_{G}$ and $p_{B}$ are the loss probabilities in the "good" and "bad" state, respectively, and $p_{G \rightarrow B}$ and $p_{G \leftarrow B}$ are the corresponding transition probabilities. We set $p_{G}=0$ and $p_{B}=0.5$, which means that on average, every second packet gets lost during bursty phases. Furthermore, $p_{G \rightarrow B}$ is set to 0.1 , which results in an average burst length of $1 / p_{G \rightarrow B}=10$. The remaining parameter $p_{G \leftarrow B}$ is used to control the expected overall amount of loss $\left(p_{G \leftarrow B} p_{G}+p_{G \rightarrow B} p_{B}\right) /\left(p_{G \rightarrow B}+p_{G \leftarrow B}\right)$ to obtain the same loss ratios as considered in Section V-C.

The simulation results are presented in Table III and Fig. 8. We expected them to be lower than those of Section V-C. More precisely, at equal average loss rates, we expected to measure a larger amount of distortion for test sequences affected by bursty loss than for sequences affected by independent loss. However, it turned out that this is not true for a small fraction of sequences: for example, $\mathcal{P}_{\mathrm{EF}}$ and $\mathcal{P}_{\mathrm{STp}}$ perform up to $0.7 \mathrm{~dB}$ better for the sequence NEWS. This phenomenon was already observed in [22], where it is correctly pointed out that increasing the average burst length does not always contribute to a larger expected distortion. We believe that this anomaly is caused by the higher proportion of static picture content in the particular sequences as the loss of multiple, temporally adjacent but highly similar regions can in most cases be well-concealed. Even more interestingly, in contrast to our results, in [22], the distortion of NEWS positively correlates with the average burst length, which suggests that the susceptibility of a stream to bursty loss not only depends on the source sequence, but also on the encoding parameters used.

$\mathcal{P}_{\mathrm{AbS}}$ and $\mathcal{P}_{\mathrm{ScMP}_{\text {fixed }}}$ provide similar results when the loss ratio is low, analogous to Section V-C. Yet at high loss ratios, $\mathcal{P}_{\mathrm{ScMP}_{\text {fixed }}}$ provides a more significant performance improvement over $\mathcal{P}_{\mathrm{AbS}}$ than when streams are affected by independent loss. As expected, the use of $\mathcal{P}_{\mathrm{ScMP}_{\text {adap }}}$ further improves the quality of reconstructed video streams, and the improvement positively correlates with the packet loss ratio. Similar to the previous section, in some cases, $\mathcal{P}_{\mathrm{ST}}$ performs worse than $\mathcal{P}_{\mathrm{EF}}$ at low loss rates, as, e.g., depicted in Fig. 8(b). The explanation for this is very likely the same as the one given in Section V-C: 


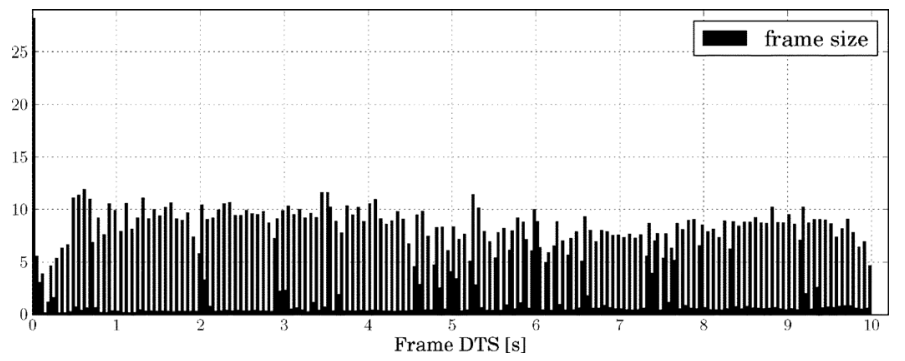

Fig. 9. Sequence CREW, encoded at $150 \mathrm{kB} / \mathrm{s}$ with periodic-intra-refresh as compared to the same sequence in Fig. 6(c), encoded without that feature.

judging media units merely based on their types is a too superficial view on their inter-dependencies.

\section{E. Low-Latency Streaming Under Loss}

So far, we considered videos which were encoded using a 16 frame closed-GOP structure having an I-frame at the beginning, which leads to a "refresh"-effect, thus stopping any error propagation at GOP boundaries. Unfortunately, I-frames are frequently more than double the size of $\mathrm{P}$-frames [cf. the spikes in Fig. 6(c)], leading to a variable bitrate, which is harder to handle for streaming systems. $x 264$ supports a feature called periodic-intra-refresh, which mitigates that problem by replacing I-frames with refresh-columns, systematically spread over all P-frames of the entire GOP. Such columns consist of intra-coded macroblocks, moving from left to right as a refresh-wave, and motion vectors in refreshed regions are restricted to only refer to also already refreshed areas, thus wiping out prediction errors originating from earlier frames. The effect is the same as when having single I-frames, but intra-coded macroblocks, which are more space-intensive (in terms of bit size) than inter-coded macroblocks, are evenly distributed across multiple frames, thus smoothing the video bitrate. To demonstrate the difference, the same test sequence of Fig. 6(c) was encoded using the same bitrate but with periodic-intra-refresh enabled. The outcome, depicted in Fig. 9, shows a smoothed video bitrate primarily due to the absence of spiky I-frames (with the exception of a large initial IDR-frame).

To investigate whether our prioritization scheme still provides accurate results in combination with streams encoded using periodic-intra-refresh, we repeated the experiments of previous sections using the same bitrates. Regarding encoding parameters, we allowed at most one consecutive B-frame, and restricted, forced by limitations of $x 264$ 's current implementation, the length of prediction lists to 1 frame. Due to these altered encoding settings and resulting steadier video bitrates, $\beta$, which determinatively influences the overall delay, can be further reduced to 6 frames. The results are depicted in Fig. 10. It is noticeable that the video quality of sequences when using the modified encoding settings is slightly lower, as can be seen by comparing PSNR values at a packet loss ratio of $0 \%$, justifiable by the lower yield in temporal redundancy reduction. Yet streams are, besides having a lower latency, in general more error resilient due to shorter dependency chains, as is apparent from the flatter curves of Fig. 10 when compared to Figs. 7 and 8. The results' pattern is similar to those of Sections V-C and V-D: both $\mathcal{P}_{\mathrm{ScMP}_{\text {fixed }}}$ and $\mathcal{P}_{\mathrm{ScMP}_{\text {adapt }}}$ lead to similar quality improvements as $\mathcal{P}_{\mathrm{AbS}}$ at low loss rates. At high loss ratios, $\mathcal{P}_{\mathrm{ScMP}_{\text {fixed }}}$ outperforms $\mathcal{P}_{\mathrm{AbS}}$, and $\mathcal{P}_{\mathrm{ScMP}_{\text {adapt }}}$ leads to further improvements in all test cases. This becomes even more evident when considering the performance curves of experiments where a Gilbert-Elliot loss generator was used [see Fig. 10(d)-(f)]. In contrast to that, $\mathcal{P}_{\mathrm{STp}}$ performs worse than in previous experiments due to its coarse grain. More specifically, because of the absence of I-slices (except for the first frame) when using periodic-intra-refresh, $\mathcal{P}_{\mathrm{ST}}$ is only able to differentiate between $\mathrm{P}$ - and B-slices, but it cannot distinguish amongst slices of the same type, e.g., by inspecting the fraction of contained refresh-macroblocks.

\section{F. Low-Latency Streaming Over a Wireless Link}

Up to now, we investigated how different prioritization schemes can improve the video quality under various loss conditions. To determine the prioritizations' benefits for a wide range of loss rates, losses were artificially created by using the loss models of dfSim and netem. In this section, we evaluate the proposed selective retransmit streaming system in an environment where the user watches a live stream over a wireless link, and where video quality impairments are exclusively caused by non-artificial packet losses.

The test environment used encompasses the server application being located at a Planetlab ${ }^{5}$ node in Norway and the wireless setup locally deployed in Austria [see Fig. 11(a)]. The latter consists of a router providing a local $802.11 \mathrm{~g}$ wireless LAN with internet access, a notebook acting as streaming client, and two additional wireless devices producing background traffic. This environment can be regarded as a common real-life situation where a user consumes multimedia content, e.g., via his/her smart phone at a public hot spot or via a laptop/mobile device within the wireless home network. The bandwidth of the link between the video server and the wireless router notably exceeded the bitrate of the video content to be transmitted, which kept packet loss at the wired side at a very low level. The latency of that link was on average $\mu=71 \mathrm{~ms}$ with a standard deviation of $\sigma=1.8 \mathrm{~ms}$. The majority of transmission failures occurred within the wireless channel, which were caused by average to poor signal strength and moderate background traffic. The latter was produced by deploying a script at the two additional wireless devices, which randomly requests websites at irregular intervals of about $5 \mathrm{~s}$. The position of these devices remained unchanged throughout all tests, and the measured signal strength was on average $-28 \mathrm{~dB}$ and $-44 \mathrm{~dB}$, which can be classified as "good" and "average" reception quality respectively. In contrast to that, we selected three different locations for the device receiving the video stream, which were characterized by an "average" $(\approx-50 \mathrm{~dB})$, a "poor" $(\approx-60 \mathrm{~dB})$, and a "very poor" $(\approx-70 \mathrm{~dB})$ reception quality.

For every test sequence and for every prioritization scheme, we triggered a streaming session with the receiver being located at one of those three locations, and we repeated that procedure $n=10$ times. By using the library $i w l i b$ [45], we continuously tracked the signal strength at the receiver throughout all

\footnotetext{
${ }^{5} \mathrm{~A}$ global network services research network; http://www.planet-lab.org/
} 


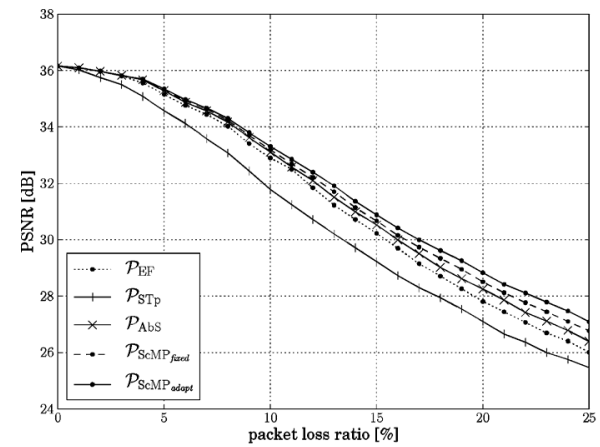

(a)

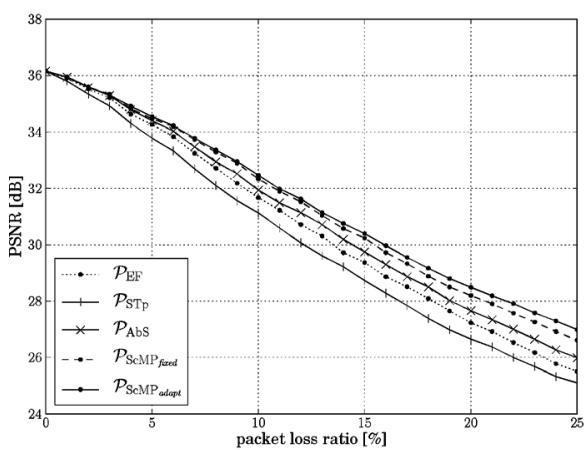

(d)

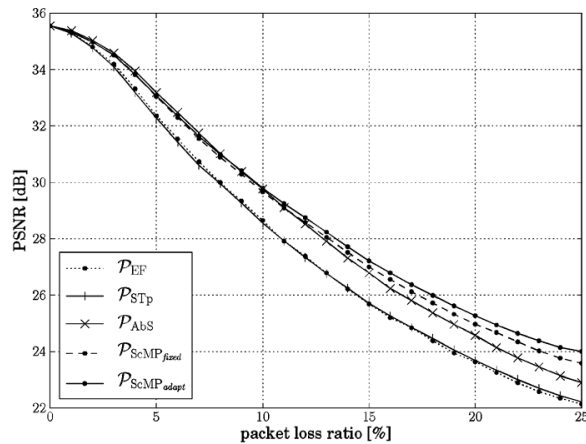

(b)

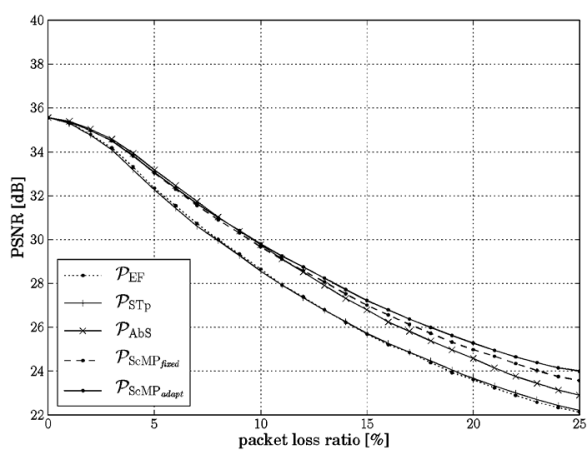

(e)

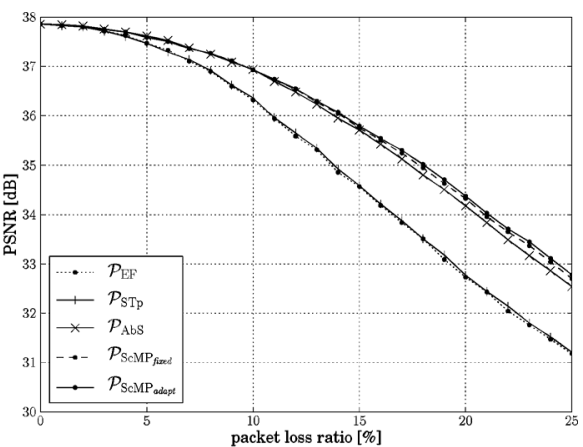

(c)

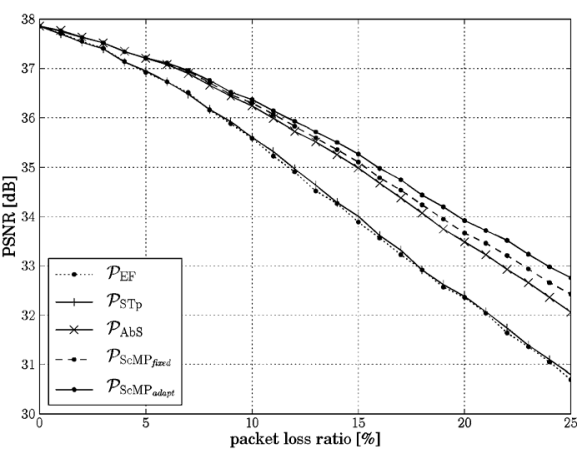

(f)

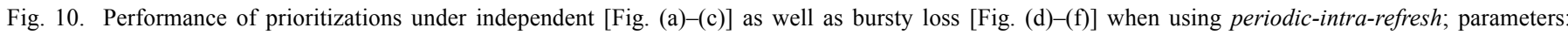
b frames $=1$, re $f=1, \beta=6$. (a) COASTGUARD. (b) MOBILE. (c) WATERFALL. (d) COASTGUARD. (e) MOBILE. (f) WATERFALL.

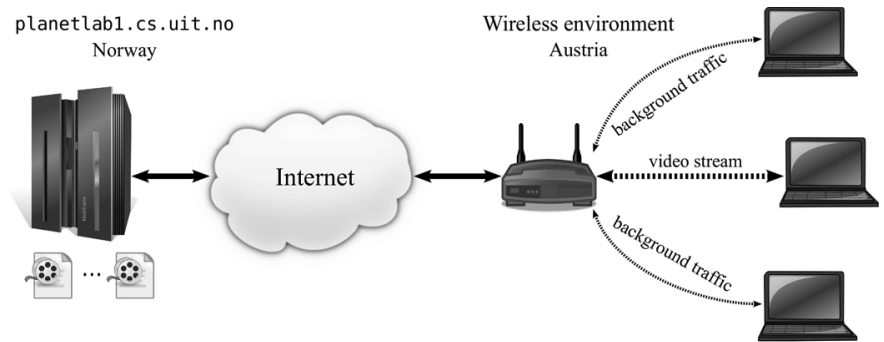

(a)

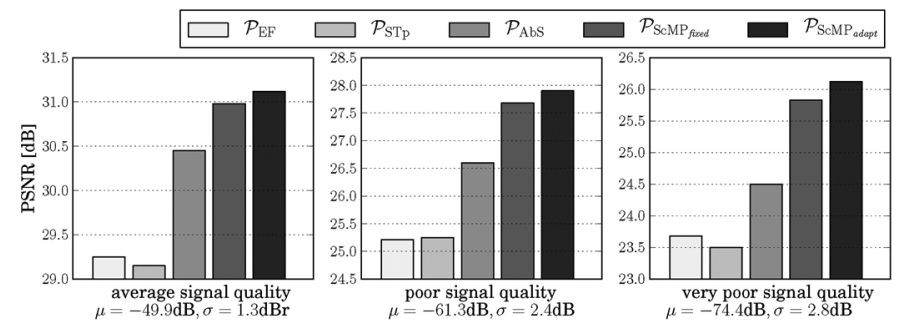

(c)

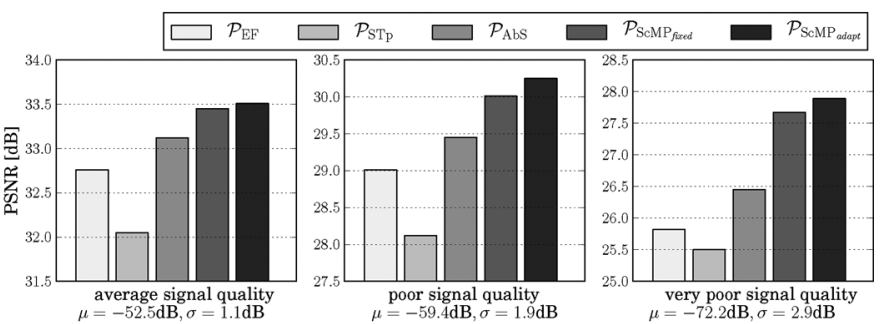

(b)

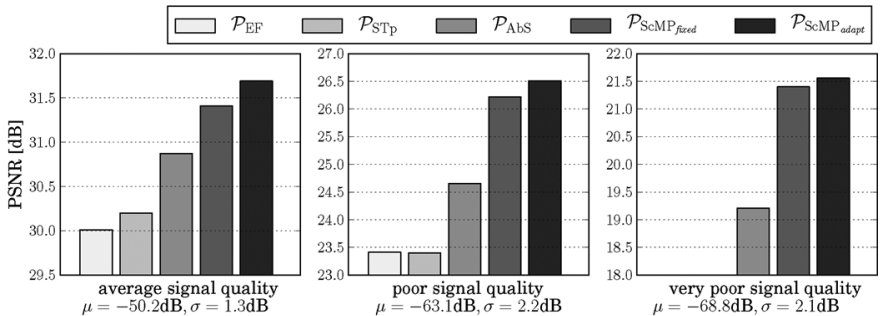

(d)

Fig. 11. Wireless test setup used in Section V-F and measurement results with sequences being encoded with periodic-intra-refresh enabled; the average video quality is shown, measured at three different locations. (a) Wireless test environment: reception of a live stream from a remote video server over a wireless link. (b) Sequence COASTGUARD, encoded at $150 \mathrm{kB} / \mathrm{s}$. (c) Sequence MOBILE, encoded at $200 \mathrm{kB} / \mathrm{s}$. (d) Sequence WATERFALL, encoded at $850 \mathrm{kB} / \mathrm{s}$.

streaming sessions. The obtained results in terms of average received video quality are depicted in Fig. 11(b)-(d). With the sequences COASTGUARD and MOBILE, the obtained streams could be entirely decoded for all runs. However, with the sequence WATERFALL, which has a higher resolution (cf. Tables II and III) and which was consequently encoded at a higher bitrate, the decoder was not able to reconstruct the video stream at the third location (very poor signal quality) when $\mathcal{P}_{\mathrm{EF}}$ or $\mathcal{P}_{\mathrm{ST}}$ was used. Besides severe packet loss, the major reason for that is the rate adaptation mechanism of the receiver's wireless network inter- face driver, ${ }^{6}$ which, based on the recent loss history, frequently reduces the maximum allowed transmission rates below WATERFALL's video bitrate.

The measured video qualities of the transmitted streams at the wireless playback device [Fig. 11(b)-(d)] show strong similarities with the results obtained in previous sections: The use of content-aware selective retransmit mechanisms employing $\Psi_{\mathrm{AbS}}, \Psi_{\mathrm{ScMP}_{\text {fixed }}}$, or $\Psi_{\mathrm{ScMP}_{\text {adapt }}}$ leads to significant

${ }^{6}$ iwlagn driver rate adaptation: http://git.kernel.org/?p=linux/kernel/git/iwlwifi/iwlwifi-2.6.git;a=blob; $\mathrm{f}=$ drivers/net/wireless/iwlwif/iwl-agn-rs.c 


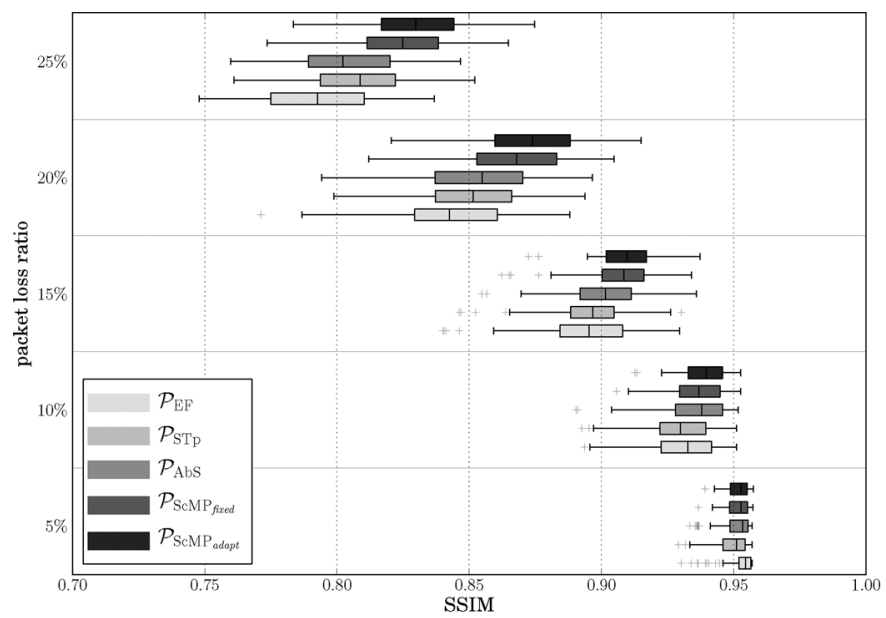

(a)

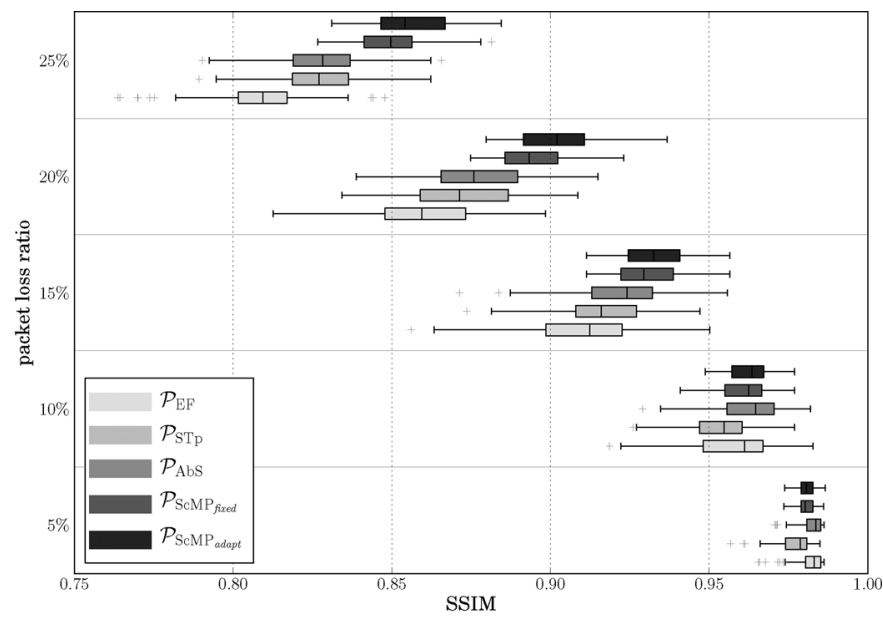

(b)

Fig. 12. Video quality measurement using the structural similarity index (SSIM); performance under bursty loss, bframes $=3$, re $f=4, \beta=10$. (a) Sequence COASTGUARD encoded at $125 \mathrm{kB} / \mathrm{s}$. (b) Sequence MOBILE encoded at $250 \mathrm{kB} / \mathrm{s}$.

video quality improvements with all three sequences. Again, $\mathcal{P}_{\text {ScMP }_{\text {fixed }}}$ outperforms $\mathcal{P}_{\mathrm{AbS}}$ (by up to $1.5 \mathrm{~dB}$ ), and the incorporation of feedback as with $\mathcal{P}_{\mathrm{ScMP}_{\text {adapt }}}$ results in further improvements.

\section{G. Roundup of Results}

In summary, $\mathcal{P}_{\mathrm{ScMP}_{\text {fixed }}}$ delivers estimates which are at low loss rates almost as accurate as those derived by $\mathcal{P}_{\mathrm{AbS}}$, independent of the loss model and encoding parameters used. This is quite remarkable when considering the fact that $\mathcal{P}_{\mathrm{ScMP}_{\text {fixed }}}$, in strong contrast to $\mathcal{P}_{\mathrm{AbS}}$, operates in real time. With growing loss rates, $\mathcal{P}_{\mathrm{ScMP}_{\text {fixed }}}$ outperforms all reference mechanisms for most test sequences, which is especially true in the presence of bursty loss. By considering feedback and adapting the sendpriority of media units according to loss experienced in the recent past, $\mathcal{P}_{\mathrm{ScMP}_{\text {ada } t}}$ can further improve the quality-of-experience (QoE) of consumers even when strict timing constraints are in place as with the streaming system considered in this work. At higher loss rates or when losses are correlated, $\mathcal{P}_{\mathrm{ScMP}_{\text {adapt }}}$ sets itself apart from all other mechanisms by incorporating receiver signalling to detect possible link breakages within temporal dependency chains, thus being able to closer estimate the situation-related relevance of pending media units.

Regarding the efficiency of $\mathcal{P}_{\mathrm{AbS}}$, we note that $\Psi_{\mathrm{AbS}}$ is derived based on the distortion in terms of MSE when the related packet is not available to the decoder. It is well known that there exists a direct relationship between MSE and PSNR, and naturally, evaluating the performance of $\mathcal{P}_{\mathrm{AbS}}$ by using the PSNR metric leads to above-average results, i.e., it casts a more favorable light on that scheme. Thus, we repeated the experiments of Fig. 8(a) and (b), and assessed the quality of the reconstructed videos by using the structural similarity index (SSIM) as proposed by Wang et al. [46]. The results, depicted in Fig. 12(a) and (b), respectively, underpin those obtained from previous measurements: Both $\mathcal{P}_{\mathrm{ScMP}_{\text {fixed }}}$ and $\mathcal{P}_{\mathrm{ScMP}_{\text {adapt }}}$ lead to substantial performance improvements over $\mathcal{P}_{\mathrm{EF}}$ and $\mathcal{P}_{\mathrm{ST} \mathrm{p}}$. As predicted, SSIM measurements of $\mathcal{P}_{\mathrm{AbS}}$ indicate a less significant gain in video quality than when PSNR is used, recognizable by the higher proximity of the box related to $\mathcal{P}_{\mathrm{AbS}}$ to those of the rather poor performing metrics $\mathcal{P}_{\mathrm{EF}}$ and $\mathcal{P}_{\mathrm{ST}}$. For instance, $\mathcal{P}_{\text {AbS }}$ performs worse than $\mathcal{P}_{\mathrm{ST}}$ in Fig. 12(a) at a loss rate of $25 \%$, which is not the case when PSNR is used [see Fig. 8(a)].

\section{CONCLUSION AND FUTURE WORK}

In this paper, we proposed a real-time-capable technique to prioritize media units (with the focus on H.264/AVC NAL units) regarding their impact on video quality, and demonstrated how such a scheme can be beneficially deployed in streaming systems supporting selective ARQ. We explained how this prioritization scheme derives estimates by analyzing the partitioning of macroblocks, the spatial extent of temporal dependencies, and the length and strength of prediction chains existing among them to track error propagation. Using this as a basis, we additionally showed how feedback can be incorporated to fine-tune priorities and to adapt the send schedule according to present network conditions. The accuracy of both static and feedbackadaptive prioritization schemes was demonstrated via simulation results, backed up by testbed measurements, and significant performance improvements over selected reference approaches could be observed in terms of both PSNR and SSIM. Further affirmative results from experiments with low-latency encoding settings in a wireless environment, paired with the ability to derive estimates in real time, render our approach especially promising for unreliable live video delivery systems supporting selective retransmissions.

As demonstrated in previous work, the use of the presented prioritization scheme is also beneficial when applied to streaming systems using forward error correction codes. Regarding the question of whether to rely on selective ARQ or unequal FEC techniques, the fields of application have to be carefully analyzed: FEC does not introduce any additional delay at the cost of an increased bandwidth consumption, and it does not need a feedback channel, thus circumventing the ACK-implosion problem in broadcast scenarios. In contrast, ARQ trades delay for bandwidth and it is known to perform superior to FEC when packet loss occurs in long bursts [47]. Additionally, applications can still make use of all received 
packets in times of severe loss whereas with FEC, a large number of packets (e.g., LT codes) or even all packets (e.g., Reed-Solomon codes) will not be decodable when their number is too small, which is a major drawback in connection with video streaming systems where error concealment mechanisms may still be able to satisfyingly reconstruct partially received frames from a subset of the original data.

Based on these considerations, future work will be devoted to the evaluation of our prioritization technique in hybrid ARQ schemes where we aim to, on the one hand, combine the advantages of FEC and ARQ and, on the other hand, given the application requirements, find a suitable tradeoff between their disadvantages. A further goal is to increasingly focus on wireless and mobile networks, and to analyze how changed conditions like higher delay, jitter, unsteady bandwidth constraints, and a higher correlation regarding loss, in comparison to wired networks, influence the effectiveness of the approach, and to detect whether further adaptations are needed to obtain optimal results. Finally, we plan to investigate whether our approach is also applicable to H.264's scalable extension SVC. Obviously, it can be applied to the base layer without changes as this forms an H.264/AVC compliant bitstream per definition, but we highly doubt that accurate results can be obtained without any modification when being applied to higher layers.

\section{REFERENCES}

[1] M. Schier and M. Welzl, "Selective packet discard in mobile video delivery based on macroblock-level distortion estimation," in Proc. IEEE Infocom MoViD, Rio de Janeiro, Brazil, Apr. 2009, pp. 1-6.

[2] L. Huo, Q. Fu, Y. Zou, and W. Gao, "Network adapted selective framedropping algorithm for streaming media," IEEE Trans. Consum. Electron., vol. 53, pp. 417-423, Mar. 2007.

[3] M. Schier and M. Welzl, "Content-aware selective reliability for DCCP video streaming," in Proc. Int. Conf. Multimedia Computing and Information Technology, Sharjah, UAE, Mar. 2010, pp. 53-56.

[4] A. Huszak and S. Imre, "Source controlled semi-reliable multimedia streaming using selective retransmission in DCCP/IP networks," Elsevier J. Comput. Commun, vol. 31, pp. 2676-2684, Jul. 2008.

[5] P. Bucciol, E. Masala, E. Filippi, and J. C. De Martin, "Cross-layer perceptual ARQ for video communications over 802.11e wireless networks," Hindawi J. Adv. Multimedia, 2007.

[6] A. Talari and N. Rahnavard, "Unequal error protection rateless coding for efficient MPEG video transmission," in Proc. IEEE MilCom Conf., Boston, MA, Oct. 2009, pp. 1-7.

[7] J. Korhonen and P. Frossard, "Flexible forward error correction codes with application to partial media data recovery," Elsevier J. Image Commun., vol. 24, pp. 229-242, Mar. 2009.

[8] A. Fiandrotti, D. Gallucci, E. Masala, and E. Magli, "Traffic prioritization of H.264/SVC video over 802.11e ad hoc wireless networks," in Proc. Int. Conf. Computer Communications and Networks, St. Thomas, U.S. Virgin Islands, Aug. 2008, pp. 1-5.

[9] E. Masala, A. Vesco, M. Baldi, and J. C. De Martin, “Optimized H.264 video encoding and packetizaton for video transmission over pipeline forwarding networks," IEEE Trans. Multimedia, vol. 11, pp. 972-985, Aug. 2009.

[10] G. Sun, W. Xing, and D. Lu, "A content-aware packets priority ordering and marking scheme for H.264 video over diffserv network," in Proc. IEEE Conf. Circuits and Systems, Macao, China, Nov. 2008, pp. $1735-1738$.

[11] M. Schier and M. Welzl, "Video streaming using content-aware unequal error protection fountain codes," in Proc. IEEE Consumer Communications and Networking Conf., Las Vegas, NV, Jan. 2011, pp. $525-526$.

[12] M. Schier and M. Welzl, "A content-aware rateless error protection scheme for live video streaming systems," in Proc. IEEE WoWMoM, Lucca, Italy, Jun. 2011.

[13] E. Gürses and G. B. Akar, "Selective frame discarding for video streaming in TCP/IP networks," in Proc. Int. Packet Video Workshop, Nantes, France, Apr. 2003.
[14] S.-H. Chang, R.-I. Chang, J.-M. Ho, and Y.-J. Oyang, "A priority selected cache algorithm for video relay in streaming applications," IEEE Trans. Broadcast., vol. 53, pp. 79-91, Mar. 2007.

[15] N. Feamster and H. Balakrishnan, "Packet loss recovery for streaming video," in Proc. Int. Packet Video Workshop, Pittsburgh, PA, Apr. 2002.

[16] W. Zhang, Q. Zheng, and Y. Lian, "Tree-aware selective frame discard for P2P IPTV system on set-top boxes," IEEE Trans. Consum. Electron., vol. 55, pp. 1982-1987, Nov. 2009.

[17] W.-T. Chen, T.-C. Lin, Y.-C. Chang, and J.-C. Chen, "Dynamic packet selection for H.264 video streaming over IEEE 802.11e WLANs," in Proc. Wireless Communications and Networking Conf., Las Vegas, NV, Mar. 2008, pp. 3133-3138.

[18] R. Zhang, S. L. Regunathan, and K. Rose, "Video coding with optimal inter/intra-mode switching for packet loss resilience," IEEE $J$. Sel. Areas Commun., vol. 18, pp. 966-976, Jun. 2000.

[19] Y. Shen, P. C. Cosman, and L. B. Milstein, "Video coding with fixed-length packetization for a tandem channel," IEEE Trans. Image Process., vol. 15, pp. 273-288, Feb. 2006.

[20] C. Schmidt and K. Rose, "First-order distortion estimation for efficient video streaming at moderate to high packet loss rates," in Proc. Int. Packet Video Workshop, Lausanne, Switzerland, Nov. 2007, pp. 318-325.

[21] H. Yang and K. Rose, "Advances in recursive per-pixel end-to-end distortion estimation for robust video coding in H.264/AVC," IEEE Trans. Circuits Syst. Video Technol., vol. 17, pp. 845-856, Jul. 2007.

[22] Z. Li, J. Chakareski, X. Niu, Y. Zhang, and W. Gu, "Modeling and analysis of distortion caused by markov-model burst packet losses in video transmission," IEEE Trans. Multimedia, vol. 19, pp. 917-931, Jul. 2009.

[23] Y. Wang, Z. Wu, and J. M. Boyce, "Modeling of transmission-lossinduced distortion in decoded video," IEEE Trans. Circuits Syst. Video Technol., vol. 16, pp. 716-732, Jun. 2006.

[24] F. Babich, M. D'Orlando, and F. Vatta, "Video quality estimation in wireless IP networks: Algorithms and applications," ACM Trans. Multimedia Comp., Commun. A, vol. 4, pp. 3:1-3:18, Feb. 2008.

[25] E. Masala and J. C. De Martin, "Analysis-by-synthesis distortion computation for rate-distortion optimized multimedia streaming," in Proc. IEEE Int. Conf. Multimedia and Expo, Baltimore, MD, Jul. 2003, vol. 3, pp. $345-348$.

[26] F. D. Vito, D. Quaglia, and J. C. De Martin, "Model-based distortion estimation for perceptual classification of video packets," in Proc. Multimedia Signal Processing Workshop, Siena, Italy, Sep. 2004, pp. 79-82.

[27] P. Bucciol, E. Masala, and J. C. De Martin, "Perceptual ARQ for H.264 video streaming over $3 \mathrm{G}$ wireless networks," in Proc. IEEE Int. Conf. Communications, Paris, France, Jun. 2004, vol. 3, pp. 1288-1292.

[28] M. Baldi, J. C. De Martin, E. Masala, and A. Vesco, "Quality-oriented video transmission with pipeline forwarding," IEEE Trans. Broadcast., vol. 54, pp. 542-556, Sep. 2008.

[29] A. Vesco, E. Masala, and C. Novara, "Distortion prediction for video quality optimization over packet switched networks," in Proc. IEEE Globecom Conf., Honolulu, HI, Nov. 2009, pp. 1-6.

[30] H.264 - Advanced Video Coding for Generic Audiovisual Services, Telecommunication Standardization Sector ITU Std., Mar. 2010.

[31] A. Yu, G. Martin, and H. Park, "Fast inter-mode selection in the H.264/AVC standard using a hierarchical decision process," IEEE Trans. Circuits Syst. Video Technol., vol. 18, pp. 186-195, Feb. 2008.

[32] Y.-H. Huang, T.-S. Ou, and H.H. Chen, "Fast decision of block size, prediction mode, and intra block for H.264 intra prediction," IEEE Trans. Circuits Syst. Video Technol., vol. 20, pp. 1122-1132, Aug. 2010.

[33] J. M. Boyce, "Weighted prediction in the H.264-AVC video coding standard," in Proc. Int. Symp. Circuits and Systems, Vancouver, BC, Canada, May 2004, vol. 3, pp. 789-792.

[34] I. Rhee and S. R. Joshi, "Error recovery for interactive video transmission over the internet," IEEE Trans. Veh. Technol., vol. 18, pp. 1033-1049, Jun. 2000.

[35] D. Vatolin, D. Kulikov, and A. Parshin, MPEG-4 AVC/H.264 Video Codecs Comparison, Moscow State University, Graphics and Media Lab, Moscow, Russia, 2010, Tech. Rep. 6.

[36] S. Khan, S. Duhovnikov, E. Steinbach, and W. Kellerer, "MOS-based multiuser multiapplication cross-layer optimization for mobile multimedia communication," Hindawi J. Adv. Multimedia, Jun. 2007, Article-id: 94918.

[37] S. Hemminger, "Netem: Emulating real networks in the lab," in Proc. Linux Conf. Australia, Apr. 2005

[38] V. Paxson and M. Allman, RFC 2988: Computing TCP's Retransmission Timer, Nov. 2000.

[39] A. C. Begen and Y. Altunbasak, "An adaptive media-aware retransmission timeout estimation method for low-delay packet video," IEEE Trans. Multimedia, vol. 9, pp. 333-347, Feb. 2007. 
[40] M. Yajnik, S. Moon, J. Kurose, and D. Towsley, "Measurement and modelling of the temporal depedence in packet loss," in Proc. IEEE Infocom Conf., New York, Mar. 1999, vol. 1, pp. 345-352.

[41] D. Loguinov and H. Radha, "End-to-end internet video traffic dynamics: Statistical study and analysis," in Proc. IEEE Infocom Conf., New York, Jun. 2002, vol. 2, pp. 723-732.

[42] K. K. Lee and S. T. Chanson, "Packet loss probability for bursty wireless real-time traffic through delay model," IEEE Trans. Veh. Technol., vol. 53, pp. 929-938, May 2004.

[43] E. N. Gilbert, "Capacity of a burst-noise channel," Bell Syst. Tech. J., vol. 39, pp. 1253-1265, Sep. 1960.

[44] E. O. Elliot, "Estimates of error rates for codes on burst-noise channels," Bell Syst. Tech. J., vol. 42, pp. 1977-1997, Sep. 1963.

[45] J. Tourrilhes, Wireless Tools for Linux. [Online]. Available: http://www.hpl.hp.com/personal/Jean Tourrilhes/Linux/Tools.html.

[46] Z. Wang, A. C. Bovik, H. R. Sheikh, and E. P. Simoncelli, "Image quality assessment: From error visibility to structural similarity," IEEE Trans. Image Process., vol. 13, pp. 600-612, Apr. 2004.

[47] M. Zorzi, "Some results on error control for burst-error channels under delay constraints," IEEE Trans. Veh. Technol., vol. 50, pp. 12-24, Jan. 2001.

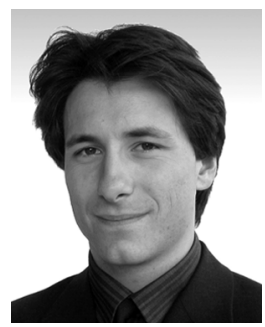

Michael Schier (A'11) received the B.Sc. degree in computer science and the Dipl.Ing. degree in computer science (both with distinction) from the University of Innsbruck, Innsbruck, Austria, in 2006 and 2008 , respectively. He is currently pursuing the Ph.D. degree.

$\mathrm{He}$ is a research assistant and member of the Distributed and Parallel Systems group at the Institute of Computer Science in Innsbruck. Previously, he was with the Research and Development Department, Besi, Inc., Radfeld, Austria, during the summers of 2004 and 2005, and with Ricmar Technology GmbH, Kramsach, Austria, in 2006 and 2007. His research interests are multimedia communications and video signal processing.

Mr. Schier received the Diploma Thesis Award from the Semiramis Research and Service Unit, Austria, in 2009, and Scholarships for Academic Excellence and a Ph.D. Scholarship from the University of Innsbruck from 2003 to 2011.

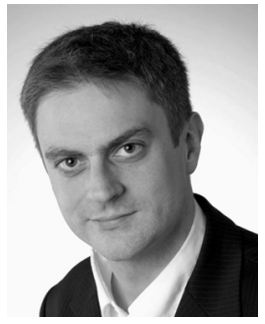

Michael Welzl (A'03) received the Ph.D. degree (with distinction) and the habilitation degree from the University of Darmstadt, Darmstadt, Germany, in 2002 and 2007, respectively.

His Ph.D. thesis "Scalable performance signalling and congestion avoidance" was published by Springer as a research monograph in 2003 and received the "Best Dissertation Award 2004" from the German GI/ITG section on Communication and Distributed Systems. His habilitation thesis, the Wiley book "Network Congestion Control: Managing Internet Traffic", is the only introductory book on network congestion control. Both books received awards from the University of Innsbruck, Innsbruck, Austria.

He spent two years as a research assistant at the Telecooperation Department, University of Linz, Linz, Austria, before joining the faculty of the newly founded Institute of Computer Science at the University of Innsbruck in 2001, where he led a research team on Network Support for Grid Computing. In May 2009, he joined the Department of Informatics of the University of Oslo as an Associate Professor. He was appointed to a Full Professorship in September 2009.

Prof. Welzl has been primary investigator of several funded research projects, coordinated the EC FP6 IST STREP project "EC-GIN", is co-chair of the IRTF Internet Congestion Control Research Group (ICCRG), and actively participates in several IETF and IRTF groups in addition to contributing to academic workshops, conferences, and journals in various roles. 